\title{
Coagulation Equations with Gelation
}

\author{
E. M. Hendriks, ${ }^{1}$ M. H. Ernst, ${ }^{1}$ and R. M. Ziff ${ }^{2,3}$
}

Received August 25, 1982; revised October 18, 1982

\begin{abstract}
Smoluchowski's equation for rapid coagulation is used to describe the kinetics of gelation, in which the coagulation kernel $K_{i j}$ models the bonding mechanism. For different classes of kernels we derive criteria for the occurrence of gelation, and obtain critical exponents in the pre- and postgelation stage in terms of the model parameters; we calculate bounds on the time of gelation $t_{c}$, and give an exact postgelation solution for the model $K_{i j}=(i j)^{\omega}(\omega>1 / 2)$ and $K_{i j}=a^{i+j}$ $(a>1)$. For the model $K_{i j}=i^{\omega}+j^{\omega}(\omega<1$, without gelation) initial solutions are given. It is argued that the kernel $K_{i j} \sim(i j)^{\omega}$ with $\omega \simeq 1-1 / d$ ( $d$ is dimensionality) effectively models the sol-gel transformation in polymerizing systems and approximately accounts for the effects of cross-linking and steric hindrance neglected in the classical theory of Flory and Stockmayer $(\omega=1)$. For all $\omega$ the exponents, $\tau=\omega+3 / 2$ and $\sigma=\omega-1 / 2, \gamma=(3 / 2-\omega) /(\omega-1 / 2)$ and $\beta=1$, characterize the size distribution, at and slightly below the gel point, under the assumption that scaling is valid.
\end{abstract}

KEY WORDS: Smoluchowski equation; coagulation; polymerization; solgel phase transition; gelation; percolation; critical exponents.

\section{INTRODUCTION}

In a recent letter ${ }^{(1)}$ we discussed a kinetic model of polymerization in which bonding between polymers takes place only at their surface, and fragmentation is absent. This model is described by the coagulation equa$\operatorname{tion}^{(2)}$

$$
\dot{c}_{k}=\frac{1}{2} \sum_{i+j=k} K_{i j} c_{i} c_{j}-c_{k} \sum_{j=1}^{\infty} K_{k j} c_{j}
$$

\footnotetext{
${ }^{1}$ Institut voor Theoretische Fysica, Rijksuniversiteit Utrecht, The Netherlands.

${ }^{2}$ Department of Mechanical Engineering, State University of New York, Stony Brook, New York 11794.

${ }^{3}$ Present address: Department of Chemical Engineering, Dow Building, University of Michigan, Ann Arbor, Michigan 48109, USA.
} 
with the coagulation kernel of the form $K_{i j}=s_{i} s_{j}$. Here $s_{k}$ is the average effective surface area of a $k$-mer, ${ }^{(1,3)}$ and $c_{k}(t)$ is the concentration of such $k$-mers at time $t$. It is assumed that $s_{k}$ has the asymptotic behavior

$$
s_{k} \sim k^{\omega} \quad(k \rightarrow \infty)
$$

where $\omega$ is a geometric exponent characterizing the surface of a $k$-mer, and depends upon the dimensionality $d$. For compact clusters this would be $\omega=1-1 / d$. The symbol " $\sim$ " means that the two quantities are asymptotically proportional while the symbol " $\simeq$ " used below denotes asymptotic equality (thus includes the constant of proportionality.

While this manuscript was in preparation some of the results of Ref. 1 and of the present paper were published by Leyvraz and Tschudi. ${ }^{(4)}$

In the classical Flory-Stockmayer theory of polymerization ${ }^{(5,6)}$ it is assumed that all unbounded sites are equally reactive, and that intramolecular bonding cannot occur. These assumptions correspond, in the limit of large clusters, to a surface area $s_{k}$ growing like the volume $k(\omega=1)$. The corresponding coagulation kernels become $K_{i j} \propto i j{ }^{(6-10,3)}$ This case is equivalent to the bond percolation problem on a Bethe lattice. In our model, by allowing only surface interactions $(\omega<1)$, both the mechanisms of steric hindrance and intramolecular bonding are effectively taken into account.

We are interested in the kinetic aspects of the gelation transition, i.e., the complete time evolution from a given initial distribution of sol particles (finite size clusters) through the gel point $t_{c}$ (where the infinite cluster first appears) towards the asymptotic stage where no sol is left. Therefore the coagulation equation (1.1) is in principle to be solved as an initial-value problem. In a kinetic description the gelation transition manifests itself through the sudden violation of mass conservation. The total sol mass, $M_{1}(t)=\sum k c_{k}(t)$, equals $M_{1}(0)=1$ before $t_{c}$, but starts to decrease for $t>t_{c}$ as the sol loses mass to the gel.

At the gel point $t_{c}$ one expects the size distribution to have asymptotically a power-law (rather than exponential) behavior:

$$
c_{k}\left(t_{c}\right) \simeq C k^{-\tau} \quad(k \rightarrow \infty)
$$

where $C$ is a positive constant. In the Flory-Stockmayer theory, for example, this behavior is attained with $\tau=5 / 2$. In our letter ${ }^{(1)}$ we discussed a general relation between $\tau$ and $\omega$, viz.,

$$
\tau=\omega+3 / 2
$$

From the extensive literature on the subject ${ }^{(2)}$ it is known that the asymptotic dependence of $K_{i j}$ on the cluster size at large $i$ and $j$ values is of crucial importance for the $k$ and $t$ dependence of the size distribution func- 
tion. ${ }^{(6,10-12)}$ For the model $K_{i j}=1$ the size distribution $c_{k}$ decays exponentially with $k$ at all $t \geqslant 0$ (no gelation). For $K_{i j}=i+j$ the distribution function approaches (1.3) with $\tau=3 / 2$ at infinite time. ${ }^{(10,11)}$ Here we consider models where gelation occurs within a finite time. The class of coagulation kernels, $K_{i j}=s_{i} s_{j}$, has been discussed in the literature. For $s_{k}=A k+B$ explicit solutions are known, ${ }^{(3-12)}$ including postgelation solutions. ${ }^{(7-9,4)}$ Before proceeding we review what is known about the existence and uniqueness of solutions to the initial-value problem (the initial solutions) of the coagulation equation:

(i) For kernels $K_{i j}$ bounded from above by $C_{i j}$ a unique solution satisfying the conditions of normalization and positivity exists in a finite time interval. ${ }^{(13)}$ The time interval in which existence has been proved does in general not extend to $t>C^{-1}$. From now on we shall absorb constants such as $C$ in the unit of time.

(ii) For kernels satisfying $K_{i j} \leqslant i+j$, which includes $K_{i j}=s_{i} s_{j} \leqslant$ $(i j)^{1 / 2}$, White ${ }^{(14)}$ showed the existence of global initial solutions, for which all moments are bounded on bounded time intervals. This excludes gelation within a finite time.

(iii) For kernels satisfying $K_{i j}=s_{i} s_{j}$, with $\lim _{k \rightarrow \infty} s_{k} / k=0$, Leyvraz and Tschudi $^{(3)}$ have established global existence of nonnegative solutions, but not their uniqueness.

(iv) For all other models proofs of global or local existence are lacking to our knowledge. We note that models $K_{i j}=s_{i} s_{j}$ with $s_{k}>k$ are unphysical in the present interpretation of the coagulation kernel, in that the effective surface area $s_{k}$ grows faster than the volume of $k$-mer.

In the following we shall tentatively assume the existence and uniqueness of solutions in all cases, including those where proofs are lacking and where the mathematical forms of coagulation kernel may be unphysical.

As far as gelation is concerned the following is known: For $K_{i j}$ $\leqslant(i j)^{1 / 2}$ gelation is excluded. ${ }^{(14,4)}$ For $K_{i j} \geqslant i j$ it cannot be true that $M_{1}(t)$ is constant for all $t \geqslant 0 .{ }^{(3)}$ For $K_{i j}=(i j)^{\omega}$ with $\omega>1 / 2$ the occurrence of gelation has been demonstrated by Leyvraz and Tschudi ${ }^{(4)}$ and the present authors. ${ }^{(1)}$

In this connection it is of interest to observe that the asymptotic form (1.3) of $c_{k}(t)$ at and past the gel point, has been obtained before by Klett ${ }^{(36)}$ and White ${ }^{(12)}$ in the related problem where monomers are produced by a source at a constant rate $a$, and agglomerate according to the collision matrix $K_{i j}=(i j)^{\omega}$. In this case the gel grows at a constant rate $a$, while the sol mass remains constant.

Once solutions of the coagulation equation are known, one can investigate the time dependence of $c_{k}(t)$ and its moments $M_{n}(t)=\sum k^{n} c_{k}(t)$, whose properties in the neighborhood of the critical point are characterized 
by critical exponents $\beta, \gamma$, and $\sigma^{(15)}$ We shall introduce these quantities together with the scaling postulate, and briefly review what is known in the context of the coagulation equation.

In the standard description of gelation ${ }^{(16-18)}$ the evolution of the system is not measured by the time $t$, but by the conversion or extent of reaction $p(t)$, and the distance from the gel point is measured as $p-p_{c}$, where $p_{c}=p\left(t_{c}\right)$. The conversion $p(t)$ is defined as the fraction of bonds that have been formed between monomers of the system at time $t$, i.e., the ratio of the actual number of bonds $\sum p_{k} c_{k}(t)$-where $p_{k}$ is the average (time-independent) number of bonds within $k$-mers - to the maximum total number of possible bonds, which is proportional to $\sum k c_{k}(0)=1$. If $p(t)$ is a smooth function of time with no irregularities present at the gel point, then

$$
p-p_{c} \sim t-t_{c}
$$

In the explicit solutions to be discussed in this paper $p(t)$ is indeed a smooth $^{4}$ function across the gel point $t_{c}$. Therefore critical exponents measured in $\left(p-p_{c}\right)$ or $\left(t-t_{c}\right)$ are identical. The quantities of interest are the weight average degree of polymerization (susceptibility) $\left(t \rightarrow t_{c}\right)$ :

$$
D P_{W}(t) \equiv M_{2}(t) / M_{1}(t) \sim\left|p-p_{c}\right|^{-\gamma} \sim\left|t-t_{c}\right|^{-\gamma}
$$

the average cluster size $k_{\xi}$ or $z$-average degree of polymerization $\left(t \rightarrow t_{c}\right)$ :

$$
k_{\xi} \sim D P_{z}(t) \equiv M_{3}(t) / M_{2}(t) \sim\left|p-p_{c}\right|^{-1 / \sigma} \sim\left|t-t_{c}\right|^{-1 / \sigma}
$$

The probability $G$ that a monomer belongs to an infinite cluster is called the gel fraction, and is nonzero only past the gel point $\left(t \downarrow t_{c}\right)$ :

$$
G(t)=M_{1}\left(t_{c}\right)-M_{1}(t) \sim\left|p-p_{c}\right|^{\beta} \sim\left|t-t_{c}\right|^{\beta}
$$

where $M_{1}(t)$ is the total (sol) mass with $M_{1}\left(t_{c}\right)=1$.

An important concept in modern theories of phase transitions is scaling. The scaling postulate states that $c_{k}(t)$ for large clusters in the close vicinity of the gel point has the form ${ }^{(16)}$

$$
c_{k}(t) \approx c_{k}\left(t_{c}\right) \Phi\left(k\left|t-t_{c}\right|^{1 / \sigma}\right) \approx k^{-\tau} \Phi\left(k\left|t-t_{c}\right|^{1 / \sigma}\right)
$$

With possibly different exponents $\tau$ and $\sigma$ and function $\Phi$ below and above the gel point. One easily verifies that the scaling postulate holds for the pregelation solution with $c_{k}(0)=\delta_{k 1}{ }^{(3)}$ in the case $K_{i j}=i j(\omega=1)$ by expanding it around $t_{c}$ and taking the large $k$ behavior. ${ }^{5}$ From the scaling

${ }^{4}$ This is in agreement with findings in computer simulations of kinetics of gelation processes. ${ }^{(19,20)}$ The coagulation mechanism of large clusters-although different in detail-is presumably modeled reasonably well by $K_{i j}=(i j)^{\omega}$.

${ }^{5}$ For the exact expression, see (3.1). The asymptotic form is $c_{k}(t) \simeq(2 \pi)^{-1 / 2} k^{-5 / 2}$ $\exp \left[-\frac{1}{2} k\left(t-t_{c}\right)^{2}\right]$ with $t_{c}=1$, so that $\sigma=1 / 2$ and $\tau=5 / 2$. 
postulate follows that the singular part of $M_{n}(t)$ as $t \rightarrow t_{c}$ behaves as

$$
M_{n}(t) \sim B_{n}\left|t_{c}-t\right|^{-(n+1-\tau) / o}
$$

where $B_{n}(n \geqslant 2)$ are positive constants. The exponents $\beta$ and $\gamma$ are given by $^{(16)}$

$$
\beta=(\tau-2) / \sigma, \quad \gamma=(3-\tau) / \sigma
$$

Since scaling is of fundamental importance, its validity should be investigated in the kinetic theories of gelation. Using heuristic arguments Leyvraz and Tschudi ${ }^{(4)}$ have established the scaling postulate for $t \uparrow t_{c}$ in the case $1 / 2<\omega \leqslant 1$ and showed that $\tau$ in the pre-gel state is still given by (1.4), and that

$$
\sigma=\omega-1 / 2
$$

This implies for the exponents in (1.11) the relation

$$
\gamma=(3 / 2-\omega) /(\omega-1 / 2), \quad \beta=1
$$

We note that $\beta$ is only defined in the postgelation stage, where the solutions of the coagulation equation do not have the scaling property (this is related to the absence of gel in the reaction equations, as we shall see later), but we shall show in Section 2.3 that $\beta$ has indeed the classical value one. For the scaling function Leyvraz and Tschudi obtained the asymptotic property

$$
\phi(x) \sim \exp \left(-C x^{\omega}\right) \quad(x \rightarrow 0)
$$

The purpose of this paper is (i) to demonstrate the validity of the relations (1.3) and (1.4) and provide some evidence for scaling, (ii) to present some new (postgelation) solutions to the coagulation equation for the models $K_{i j} \sim(i j)^{\omega}$ and $K_{i j} \sim a^{i+j}$, and (iii) to investigate whether some classes of coagulations kernels yield a gelation transition. Our derivation of (1.3) for the model $K_{i j} \sim(i j)^{\omega}$ (see Section 2) employs the generating function $g(x, t)$ defined as $\sum c_{k}(t) \exp (k x)$, and we determine the singularities in this function close to the origin as $t \downarrow t_{c}$. From these the dominant behavior (1.3) and higher asymptotic corrections can be determined straightforwardly. The amplitudes in the asymptotic expressions for $c_{k}(t)$ depend upon the unknown gelation rate, $-\dot{M}_{1}(t)$, which in turn depends on details of the initial size distribution, $c_{k}(0)$. In order to demonstrate that (1.3) represents a viable solution we explicitly derive (in Section 3) a special postgelation solution of the simple form [1]

$$
c_{k}(t)=c_{k}\left(t_{c}\right)\left[1+b\left(t-t_{c}\right)\right]^{-1} \quad\left(t \geqslant t_{c}\right)
$$

where $b$ is a constant, to be determined, and $t_{c}$ is the gel point (not determinable). For special cases (see Section 4) we calculate $c_{k}\left(t_{c}\right)$ and $b$ analytically $\left(s_{k}=k^{2}, s_{k}=a^{k-1}\right.$, and $s_{k}=k^{\omega}$ at large $\left.\omega\right)$, or numerically 
$\left(s_{k}=k^{\omega}\right.$ for $\left.\omega>1 / 2\right)$. In Ref. 4 existence arguments for such solutions have been given. Some evidence for the validity of the scaling postulate is given in Section 5, but two counterexamples $\left(s_{k}=k^{2}\right.$ and $\left.s_{k}=a^{k-1}\right)$ show that the scaling postulate cannot be valid for general coagulation kernels $K_{i j}=s_{i} s_{j}$. In Section 5 we also give the negative answer to the question, posed by Leyvraz and Tschudi ${ }^{(4)}$ whether the special postgelation solution (1.15) is reached from monodisperse initial conditions, $c_{k}(0)=\delta_{k 1}$. Finally we discuss (in Section 5) the Taylor series expansion of $c_{k}(t)$ around $t=0$. In Section 6 we investigate the possibility of a gelation transition for the kernels $K_{i j}=s_{i}+s_{j}$ and $K_{i j}=s_{i} r_{j}+r_{i} s_{j}$, and in Appendix B we present the explicit solution of the initial-value problem for the coagulation equation with $K_{i j}=i^{\omega}+j^{\omega}(\omega \leqslant 1)$, for which no gelation occurs. In the conclusion we compare our results with those of lattice percolation theories, and give some further comments and outlook.

\section{EXPONENTS AND AMPLITUDES IN THE POSTGELATION STAGE}

\subsection{Exponent $\tau$}

The exponent relation (1.4) can be derived in general as follows: multiply (1.1) by $e^{k x}$ and sum over all $k$, to find

$$
\dot{g}=\frac{1}{2} f^{2}-S_{0} f
$$

The dot denotes time differentiation. The generating functions are defined as

$$
\begin{aligned}
& g(x, t)=\sum_{k=1}^{\infty} c_{k}(t) e^{k x} \\
& f(x, t)=\sum_{k=1}^{\infty} s_{k} c_{k}(t) e^{k x}
\end{aligned}
$$

with

$$
S_{0}(t)=f(0, t)=\sum_{k=1}^{\infty} s_{k} c_{k}(t)
$$

We note that the $k$-summations do not include the infinite cluster. By convention we always choose $s_{1}=1$, which is possible by a redefinition of the unit of time. If we write the functions $g$ and $f$ as

$$
\begin{aligned}
& g(x, t)=M_{0}+x M_{1}+\Delta(x, t) \\
& f(x, t)=S_{0}+\delta(x, t)
\end{aligned}
$$


then $\Delta=o(x)$ and $\delta=o(1)$ for small (negative) $x$, since the moments $M_{0}$ and $M_{1}$ must exist (the mass is finite) and $S_{0}$ must exist, so that the kinetic equation (1.1) is defined. If $s_{k} \sim k^{\omega}$ for large $k$, then the existence of $S_{0}$ implies also the existence of all moments $M_{n}(t)$ with $n \leqslant \omega$, where

$$
M_{n}(t)=\sum_{k=1}^{\infty} k^{n} c_{k}(t) \quad(n=0,1, \ldots)
$$

It follows from (2.1), by setting $x=0$, that $M_{0}$ and $S_{0}$ are related by

$$
\dot{g}(0, t)=\dot{M}_{0}=-\frac{1}{2} S_{0}^{2}
$$

Furthermore (2.1) implies

$$
f=S_{0}-\left(S_{0}^{2}+2 \dot{g}\right)^{1 / 2}
$$

which can be written in the equivalent form

$$
f(x, t) / f(0, t)=1-[1-\dot{g}(x, t) / \dot{g}(0, t)]^{1 / 2}
$$

by virtue of (2.6).

To discuss the solution at and past the gel point $t_{c}$ it is necessary to look for solutions in which $M_{1}$ (representing the total mass of the sol) depends upon time (note that $M_{1}$ is a constant before the gel point). Consider first the function $f(x, t)$ for small $x$; it follows from (2.4), (2.6), and $(2.7 \mathrm{a})$ that

$$
\begin{aligned}
f & =S_{0}-\left[2 x \dot{M}_{1}(t)+\dot{\Delta}(x, t)\right]^{1 / 2} \\
& =S_{0}-\left(-2 \dot{M}_{1}\right)^{1 / 2}(-x)^{1 / 2}-\left(-2 \dot{M}_{1}\right)^{-1 / 2}(-x)^{-1 / 2} \dot{\Delta}+\cdots
\end{aligned}
$$

where both $\dot{M}_{1}$ and $x$ are negative. As might be expected past the gel point, $f$ is nonanalytic about $x=0$. A singularity in $x=0$ in the generating function (2.2) implies an algebraic tail in the expansion coefficients $s_{k} c_{k}$ for large $k$, as discussed in Appendix A. The leading singularity $(-x)^{1 / 2}$ in (2.8) yields according to (2.2) and (A.4) of Appendix A

$$
s_{k} c_{k} \simeq\left(-\dot{M}_{1} / 2 \pi\right)^{1 / 2} k^{-3 / 2} \quad(k \rightarrow \infty)
$$

Assuming $s_{k}$ has the behavior given in (1.2), it follows that

$$
c_{k} \simeq\left(-\dot{M}_{1} / 2 \pi\right)^{1 / 2} k^{-3 / 2-\omega} \quad(k \rightarrow \infty)
$$

Thus we have proved the scaling relation (1.4) and have found an expression for the amplitude $C$ in (1.3).

The behavior of $s_{k} c_{k}$ given by (2.10) is consistent with the requirement that $S_{0}$ exists for all $\omega$. However, for $M_{1}$ to be finite, we must have $\omega>1 / 2$ on account of (2.10). Only for $\omega>1 / 2$ can the kinetic equation (1.1) have solutions with time-dependent $M_{1}$. 
When $1 / s_{k}$ can be written as a Laplace transform:

$$
1 / s_{k}=\int_{0}^{\infty} d y \sigma(y) e^{-k y}
$$

$f$ and $g$ are related by

$$
g(x, t)=\int_{0}^{\infty} d y \sigma(y) f(x-y, t)
$$

and (2.1) can be transformed into an integral equation:

$$
\frac{1}{2} f^{2}(x, t)-S_{0} f(x, t)=\int_{0}^{\infty} d y \sigma(y) \dot{f}(x-y, t)
$$

The function $f(x, t)$ is subject to the boundary conditions $f(0, t)=S_{0}(t)$ and

$$
M_{1}(t)=g^{\prime}(0, t)=\int_{0}^{\infty} d y \sigma(y) f^{\prime}(-y, t)<\infty
$$

where primes denote derivatives with respect to the first variable. An expression for $S_{0}(t)$ follows from (2.13) by taking the limit $x \uparrow 0$. A specific example for $\sigma(y)$ corresponding to $s_{k}=k^{\omega}$ is

$$
\sigma(y)=y^{\omega-1} / \Gamma(\omega)
$$

Of course, when $\omega$ is a positive integer, $f$ and $g$ are related simply by differentiation:

$$
f(x, t)=\left(\frac{\partial}{\partial x}\right)^{\omega} g(x, t)
$$

\subsection{Higher-Order Corrections}

In this section we construct higher-order terms in the asymptotic expansion of $c_{k}$. Here we must assume either that $s_{k}$ equals $k^{\omega}$ exactly for all $k$, or at least that $s_{k} \sim k^{\omega}$ as $k \rightarrow \infty$ with the relative order of the next asymptotic term of $s_{k}$ lower than the relative order of the correction term as given below. We calculate the leading singularity $\Delta(x, t)$ in $g(x, t)$ from the leading singularity $(-x)^{1 / 2}$ of $f(x, t)$ in (2.8) using Eqs. (2.12) and (2.15). Since the zeroth and first derivatives of $g(x, t)$ at $x=0$ in (2.12) exist, $\left(M_{0}\right.$ and $M_{1}$ exist), $\Delta(x, t)$ in (2.4) can be written as

$$
\begin{aligned}
\Delta(x, t) & =[\Gamma(\omega)]^{-1} \int_{0}^{\infty} d y y^{\omega-1}\left[f(x-y, t)-f(-y, t)-x f^{\prime}(-y, t)\right] \\
& =x^{2}[\Gamma(\omega)]^{-1} \int_{0}^{\infty} d y y^{\omega-1} \int_{0}^{1} d \tau(1-\tau) f^{\prime \prime}(-y+\tau x, t)
\end{aligned}
$$

where primes denote derivatives with respect to the first variable. On the 
second line of (2.17) the formula ${ }^{(21)}$ for the remainder $R_{2}$ in the Taylor expansion has been used. We divide the $y$-integration interval into $\left(0, x_{0}\right)$ and $\left(x_{0}, \infty\right)$, where $x_{0}$ is some small positive constant. Since the integrand in (2.17) falls off as $e^{-y}$ for large $y$, the interval $\left(x_{0}, \infty\right)$ contributes to $\Delta$ a term of $O\left(x^{2}\right)$ for small $x$, the coefficient of which cannot be determined. If $x_{0}$ is chosen sufficiently small, the integrand in the interval $\left(0, x_{0}\right)$ can be represented by the leading term in (2.8). By finally introducing the integration variable $z$ as $y=|x| \tau x$, we obtain

$$
\begin{aligned}
\Delta(x, t) \simeq[ & {[4 \Gamma(\omega)]^{-1}\left(-2 \dot{M}_{1}\right)^{1 / 2}(-x)^{\omega+1 / 2} \int_{0}^{1} d \tau(1-\tau) \tau^{\omega-3 / 2} } \\
& \times \int_{0}^{x_{0} /|x| \tau} d z z^{\omega-1}(z+1)^{-3 / 2}+O\left(x^{2}\right)
\end{aligned}
$$

This yields to leading order

$$
\begin{aligned}
\Delta(x, t) & \simeq[4 \Gamma(\omega)]^{-1}\left(-2 \dot{M}_{1}\right)^{1 / 2}(-x)^{\omega+1 / 2} B(2, \omega-1 / 2) B(\omega, 3 / 2-\omega) \\
& =\left(-\dot{M}_{1} / 2 \pi\right)^{1 / 2} \Gamma(-1 / 2-\omega)(-x)^{\omega+1 / 2}+O\left(x^{2}\right)
\end{aligned}
$$

where $B(x, y)=\Gamma(x) \Gamma(y) / \Gamma(x+y)$. The convergence of the $z$ integral in (2.18) at large $z$ requires $\omega<3 / 2$. If $3 / 2<\omega<5 / 2$ the $z$ integral can be written as

$$
\int_{0}^{x_{0} /|x| \tau} d z z^{\omega-1}\left[(z+1)^{-3 / 2}-z^{-3 / 2}\right]^{1 / 2}+(\omega-3 / 2)^{-1}\left(x_{0} /|x| \tau\right)^{\omega-3 / 2}
$$

The last term contributes to $O\left(x^{2}\right)$ in (2.18). The expression [ $\left.\cdots\right]$ can be replaced by the remainder $R_{1}$ of the Taylor series, and the resulting integrals can be evaluated as in (2.18). The leading singularity in (2.18) is again given by (2.19), where $O\left(x^{\omega+1 / 2}\right)$ is now smaller than $O\left(x^{2}\right)$. Notice that this method does not enable us to find the complete small- $x$ behavior of $\Delta(x, t)$, but only the singular terms, which determine the asymptotic $k$ dependence of the size distribution $c_{k}$. The regular terms in $\Delta(x)$ with integral power of $x$ depend on $f$ values over the whole range of $x$ values. For larger $\omega$ values one has to subtract more regular terms than was done in (2.20), but the leading small $x$ singularity is still given by (2.19).

From $\Delta(x, t)$, given in (2.19), we can immediately derive the next term in asymptotic expansion for the case $1 / 2<\omega<3 / 2$, using (2.8) and (A.4):

$$
\begin{aligned}
c_{k}(t) \simeq & \left(-\dot{M}_{1} / 2 \pi\right)^{1 / 2} k^{-3 / 2-\omega} \\
& -\left(\ddot{M}_{1} / 4 \sqrt{\pi} \dot{M}_{1}\right)\left[\Gamma\left(-\frac{1}{2}-\omega\right) / \Gamma(-\omega)\right] k^{-2 \omega-1}+\cdots
\end{aligned}
$$

For the case $3 / 2<\omega<5 / 2$ the moment $M_{2}$ exists and $\Delta(x, t) \cong \frac{1}{2} x^{2} M_{2}+$ 
$O\left(x^{\omega+1 / 2}\right)$, and the asymptotic expansion has the form

$$
c_{k}(t) \simeq\left(-\dot{M}_{1} / 2 \pi\right)^{1 / 2} k^{-3 / 2-\omega}-(3 / 8) \dot{M}_{2}\left(-2 \pi \dot{M}_{1}\right)^{-1 / 2} k^{-5 / 2-\omega}+\cdots
$$

Next consider the case $\omega=3 / 2$, where the integral (2.18) contains a logarithmic singularity:

$$
\Delta(x, t) \simeq-\frac{1}{2}\left(-\dot{M}_{1} / 2 \pi\right)^{1 / 2} x^{2} \log (-x)+O\left(x^{2}\right)
$$

This yields according to (A.7)

$$
c_{k}(t) \simeq\left(-\dot{M}_{1} / 2 \pi\right)^{1 / 2} k^{-3}+\cdots \quad(k \gg 1)
$$

Again it is easy to obtain the next term in the asymptotic expansion by inserting (2.23) into (2.8) and using (A.6) and (A.7) with $\alpha=3 / 2$ :

$$
c_{k}(t) \simeq\left(-\dot{M}_{1} / 2 \pi\right)^{1 / 2} k^{-3}-\left[3 \ddot{M}_{1} /\left(32 \pi \dot{M}_{1}\right)\right] k^{-4} \log k+\cdots
$$

The coefficient of the term $\sim k^{-4}$ in (2.25) cannot be determined, as it contains the unknown $O\left(x^{2}\right)$-term in (2.23).

In principle one can continue this procedure to obtain higher-order $k$-corrections or results for higher $\omega$-values. In the latter case (2.22) remains valid for all $\omega>3 / 2$, but its derivation is somewhat more complicated because of the appearance of intermediate regular terms $O\left(x^{n}\right)$ with $1<n \leqslant[\omega+1 / 2]$. In the former case, when $n_{0}-1 / 2<\omega<n_{0}+1 / 2\left(n_{0}\right.$ is an integer), $c_{k}$ has the general form for $k \gg n_{0}$ :

$$
c_{k} \simeq \sum_{n=1}^{n_{0}} b_{n} k^{-n-\omega-1 / 2}+B_{\omega} k^{-2 \omega-1}+\cdots
$$

For example, in the region $1 / 2<\omega<3 / 2$ the result of the repeated iteration procedure becomes

$$
c_{k}(t) \simeq \sum_{n=0}^{\infty}\left\{\prod_{l=1}^{n} \frac{\Gamma(-1-l s)}{\Gamma\left(-\frac{1}{2}-l s\right)}\right\}\left(\frac{1}{q} \frac{d}{d t}\right)^{n}\left[\frac{q}{\Gamma(-1 / 2)}\right] k^{-\omega 3 / 2-n s}
$$

where $s=\omega-1 / 2$, the product $\prod_{l}^{n} \equiv 1$ for $n=0$, and $q=-\left(2 \dot{M}_{1}\right)^{1 / 2}$. In the classical limit $\omega \rightarrow 1$ (random percolation on a Bethe lattice ${ }^{(3,8,10)}$ ), all terms with odd $n$ vanish, and the large- $k$ expansion of the classical postgel solution [see (3.1), where $M_{1}(t)=t^{-1}$ for monodisperse initial conditions] is recovered. We note that Eq. (2.26b) describes the complete large- $k$ behavior of the size distribution for kernels asymptotically behaving as $(i j)^{\omega}$, $1 / 2<\omega<3 / 2$, i.e., it constitutes the relevant part of the solution of the coagulation equation for that case. The function $q(t)$, though, is in general 
undetermined. For (2.26b) to be valid to all orders, it is necessary that $s_{k}=k^{\omega}$ exactly.

\subsection{Critical Exponents Involving Time}

In order to determine the exponents $\beta, \gamma$, and $\sigma$ in (1.6)-(1.8) we have to know the explicit time dependence of the growth rate of the gel fraction, - $\dot{M}_{1}(t)$, since it appears in the asymptotic postgelation behavior (2.10). Unfortunately, we have only found the special postgelation solution (1.15) in explicit form, for which $M_{1}(t)=\left[1+b\left(t-t_{c}\right)\right]^{-1}$. Exponents, however, are believed to be the same for all physically acceptable size distributions. We will therefore attempt to determine the above exponents from this special solution. Close to the gel point we see that the gel fraction (1.8) behaves as $\left[\dot{M}_{1}\left(t_{c}^{+}\right)=-b\right.$ finite $]$

$$
G(t)=1-M_{1}(t) \simeq b\left(t-t_{c}\right)
$$

implying $\beta=1$ (the same as in the classical Flory-Stockmayer theory, where $s_{k} \sim k$ ). However, the exponents $\gamma$ and $\sigma$ are undefined for the physically relevant models with $s_{k} \leqslant C k$. The reason is that $M_{2}(t)$ and $M_{3}(t)$ do not exist for $t \geqslant t_{c}$, as follows from the asymptotic expression (2.10). In the other cases, where $s_{k}$ increases sufficiently fast, the relevant moments exist, but then $\mathrm{DP}(t)$ in (1.6)-(1.7) equals $\mathrm{DP}\left(t_{c}\right)$ for all $t \geqslant t_{c}$, at least in the special postgelation solution (1.15)!

What is the reason for this peculiar postgelation behavior? Past the gel point the kinetic equation (1.1) represents a system in which sol and gel do not interact. This is so because the gel particles are not present in the kinetic equation (1.1). The same holds for Stockmayer's classical theory. However, in most physical situations one would expect the sol and gel to interact. Then our kinetic equation would have to be modified by adding appropriate interactions between sol and gel particles. This has been done in the classical theory by Ziff and Stell. ${ }^{(9)}$ The latter authors show that the time dependence of the postgel solutions is strongly affected by the assumed sol-gel interactions. Among others they discuss sol-gel interactions that leads to Flory's classical postgelation solution, for which the exponents $\gamma$ and $\sigma$ are well defined, and equal to their pregelation value $\gamma=1$ and $\sigma=1 / 2$, while $\beta$ keeps the value 1 .

Adding sol-gel interaction terms to our kinetic equation may possibly change the time dependence of the gel fraction. However, we have not succeeded in finding postgelation solutions when the equation is modified in such a manner.

Another effect of the absence of sol-gel interactions is the breakdown of scaling in the postgelation stage. As we have seen below (1.9) scaling 
holds for the pregelation solution of the classical kinetic equation with $s_{k} \sim k$. Addition of appropriate sol-gel interaction terms ${ }^{(9)}$ to the classical equation yields Flory's postgel solution, which has the same analytical form as the pregel solution. Hence, in Flory's classical theory scaling holds in the pre- and postgelation stage and yields the same exponents on both sides of the phase transition. However, the scaling postulate $(1.19)$ is not valid for Stockmayer's classical postgel solution, which is of the form (1.15), nor does it hold for our special postgelation solution (1.15). In summary: the postgelation exponent $\tau=\omega+3 / 2$, found in this section, is expected to be generally valid at (and slightly below) $t_{c}$. As already argued in Ref. 1, the $k$ dependence of $c_{k}\left(t_{c}\right)$ cannot depend on the assumed interaction between sol and gel since no gel is present at $t_{c}$. The exponent $\beta$, as calculated from our special postgel solution, has its classical value one. Although in general the time dependence of the gel fraction, (1.8), may strongly depend on the assumed sol-gel interaction, its initial growth rate $\dot{M}_{1}\left(t_{c}\right)$ is not expected to do so, since at $t_{c}$ no gel is present. In addition, if the scaling form (1.9) could be continued past $t_{c}$ by adding appropriate sol-gel interactions, the scaling relation (1.11) would also predict $\beta=1$.

\section{EXPLICIT POST-GELATION SOLUTIONS}

In this section we derive an explicit postgel solution of (1.1). This solution was suggested by the case $s_{k}=k$ for which the solution in the case of monodisperse initial conditions $c_{k}(0)=\delta_{k 1}$ is known explicitly and given by ${ }^{(2,3,13)}$

$$
c_{k}(t)=t^{k-1} e^{-k t} k^{k-2} / k !
$$

for $0<t<1$, while past the gel point (at $t_{c}=1$ ) the solution is simply given by $^{(3)}$

$$
c_{k}(t)=c_{k}\left(t_{c}\right) / t
$$

Up to the gel point the total mass is conserved, hence $M_{1}\left(t_{c}\right)=1$, and past the gel point we have $M_{1}(t)=1 / t$. The inverse linear time dependence of (3.2) reflects a general scaling property of (1.1). We therefore consider a possible exact postgel solution of (1.1) of the form

$$
c_{k}(t)=c_{k}\left(t_{c}\right)\left[1+b\left(t-t_{0}\right)\right]^{-1} \quad\left(t>t_{0}\right)
$$

where $b$ is a constant, to be determined, and $t_{c}$ is the gel point (not determinable), and where the $c_{k}$ are further assumed to be normalized such that

$$
M_{1}\left(t_{c}\right)=\sum_{k=1}^{\infty} k c_{k}\left(t_{c}\right)=1
$$


On substituting (3.3) into (1.1) we find that the time part cancels and what remains is a recursion relation for the $c_{k}\left(t_{c}\right)$ :

$$
-b c_{k}\left(t_{c}\right)=\frac{1}{2} \sum_{i+j=k} s_{i} s_{j} c_{i}\left(t_{c}\right) c_{j}\left(t_{c}\right)-s_{k} c_{k}\left(t_{c}\right) \sum_{j=1}^{\infty} s_{j} c_{j}\left(t_{c}\right)
$$

The purpose of the present section is to determine the quantities $b$ and $c_{k}\left(t_{c}\right)$ explicitly. An expression for $b$ follows by letting $k=1$ (assuming $\left.c_{1} \neq 0\right)$ :

$$
b=\sum_{k=1}^{\infty} s_{k} c_{k}\left(t_{c}\right)=S_{0}\left(t_{c}\right)
$$

Then (3.5) may be written as

$$
b\left(s_{k}-1\right) c_{k}\left(t_{c}\right)=\frac{1}{2} \sum_{i+j=k} s_{i} s_{j} c_{i}\left(t_{c}\right) c_{j}\left(t_{c}\right)
$$

It is convenient to eliminate $b$ by introducing:

$$
n_{k} \equiv c_{k}\left(t_{c}\right) / b
$$

These $n_{k}$ satisfy the recursion relation

$$
\left(s_{k}-1\right) n_{k}=\frac{1}{2} \sum_{i+j=k} s_{i} s_{j} n_{i} n_{j}
$$

It has to be solved subject to the condition

$$
\sum_{k} s_{k} n_{k}=1
$$

on account of (3.6), whereas (3.4) is used to determine $b$ :

$$
\sum_{k} k n_{k}=1 / b
$$

Summing (3.9) over all $k$, one finds the relation

$$
\sum_{k} n_{k}=\frac{1}{2}
$$

Equations (3.6)-(3.12) imply $b=S_{0}\left(t_{c}\right)=2 M_{0}\left(t_{c}\right)$, where $S_{0}\left(t_{c}\right)=M_{\omega}\left(t_{c}\right)$ for the special case $s_{k}=k^{\omega}$.

For a given $n_{1}$ the recursion relation (3.9) determines all $n_{2}, n_{3} \ldots ; n_{1}$ is chosen such that (3.10) or (3.12) is satisfied. If (3.11), which determines $b$, exists, then the expression (3.3) represents a valid solution to the kinetic equation (1.1), as described below.

Consider the formulation in terms of generating functions. Equation (3.9) is equivalent to

$$
F-G=\frac{1}{2} F^{2}
$$


or

$$
F=1-(1-2 G)^{1 / 2}
$$

where the generating functions

$$
\begin{aligned}
& F(x)=\sum_{k} s_{k} n_{k} e^{k x} \\
& G(x)=\sum_{k} n_{k} e^{k x}
\end{aligned}
$$

are according to (2.12) related by

$$
G(x)=\int_{0}^{\infty} d y \sigma(y) F(x-y)
$$

The equations (3.13) and (3.15) have to be solved subject to the restriction:

$$
F(0)=1 \text { or } \quad G(0)=1 / 2
$$

as follows from (3.10) and (3.12), and $b$ is obtained via (3.11) as

$$
b^{-1}=G^{\prime}(0)
$$

Finally, combination of (3.15) and (3.13a) yields an integral equation for $F(x)$ of the form

$$
F(x)-\frac{1}{2} F^{2}(x)=\int_{0}^{\infty} d y \sigma(y) F(x-y)
$$

The previous equations (3.13)-(3.18) may be obtained directly from the corresponding equations in Section 2.1, where $G(x)=g\left(x, t_{c}\right) / 2 g\left(0, t_{c}\right)$ and $F(x)=f\left(x, t_{c}\right) / f\left(0, t_{c}\right)$, with the help of (3.3), (3.10), and (3.12).

Solving integral equation (3.18) with the restriction (3.16) is equivalent to solving the recursion relations (3.9) together with (3.12).

For small $x$ the function $F(x)$ has a singularity of the form

$$
F(x) \simeq 1-(-2 x / b)^{1 / 2}+\cdots
$$

as follows directly from (3.13b), (3.16), and (3.17), or from the integral equation (3.18), using (3.15)-(3.17). The behavior of $F$ in (3.19) implies that $n_{k} \sim k^{-\omega-1 / 2}$, when $s_{k}$ satisfies (1.2). Thus, when $\omega \leqslant 1 / 2$, the solution (3.3) is not valid, because (3.11) diverges and $b$ does not exist.

The analysis of Section 2 can of course be used to find the asymptotic $h$ dependence of $c_{k}$ for this solution. As a consequence of (3.3) all terms in (2.21), (2.22), (2.25), and (2.26) depend on time as $\left[1+b\left(t-t_{c}\right)\right]^{-1}$, so that 
these equations can be used at $t=t_{c}$ with

$$
\begin{aligned}
& \dot{M}_{1}\left(t_{c}\right)=-b M_{1}\left(t_{c}\right)=-b \\
& \ddot{M}_{1}\left(t_{c}\right)=2 b^{2} \\
& \dot{M}_{n}\left(t_{c}\right)=-b M_{n}\left(t_{c}\right)
\end{aligned}
$$

The last equation is only valid for $n<\omega+1 / 2$ since $M_{n}\left(t_{c}\right) \rightarrow \infty$ for $n \geqslant \omega+1 / 2$. All amplitudes depend on the unknown constant $b$.

Equation (3.13) or (3.18) reduces to a differential equation if $\omega$ is an integer. For $\omega=1$ (or $s_{k}=k$ ) $F$ and $G$ are simply related as $F(x)=G^{\prime}(x)$, and by differentiating (3.13a) with respect to $x$, one finds

$$
F^{\prime}-F=F F^{\prime}
$$

Its solution is $F \exp (-F)=\alpha e^{x}=e^{x-1}$, where the integration constant $\alpha$ is fixed by (3.16), and $b$ follows directly from (3.11) and (3.14) as $b^{-1}=\sum k n_{k}$ $=1$. The Lagrange expansion ${ }^{(21)}$ may be used to find $F$ as a series in powers of $e^{x}$ with the result $n_{k}=c_{k}\left(t_{c}\right)=e^{-k} k^{k-2} / k !$, in agreement with the well-known result (3.1) for the monodisperse initial condition at the gel point $t_{c}=1$. Of course, the arguments used in this section give no evidence that this solution corresponds to monodisperse initial conditions for general $\omega$.

In the next section we study several special choices of $s_{k}$ for which new solutions will be obtained.

\section{SPECIAL CASES}

\subsection{The Case $s_{k}=k^{2}$}

Here $F$ and $G$ in (3.14) are related as $F=G^{\prime \prime}$. Thus (3.13b) reduces to a differential equation:

$$
G^{\prime \prime}=1-(1-2 G)^{1 / 2}
$$

which can be integrated, after multiplying by $G^{\prime}$, to yield

$$
\frac{1}{2}\left(G^{\prime}\right)^{2}=G+\frac{1}{3}(1-2 G)^{3 / 2}-\frac{1}{3}
$$

The constant of integration $(1 / 3)$ is determined by the requirement that $G^{\prime} \rightarrow 0$, when $G \rightarrow 0(x \rightarrow-\infty)$. Introducing $F$, defined in (3.13b), one finds

$$
F^{\prime}=F(1-2 F / 3)^{1 / 2}(1-F)^{-1}
$$

which may be integrated to yield

$$
x=\int_{1}^{F} d t t^{-1}(1-t)(1-2 t / 3)^{-1 / 2}
$$


the lower limit reflecting $F(0)=1$. The integral in (4.4) may be evaluated through the substitution $u=(3-2 t)^{1 / 2}$ with the result

$$
e^{x}=\frac{\sqrt{3}+1}{\sqrt{3}-1} \frac{\sqrt{3}-r}{\sqrt{3}+r} e^{\sqrt{3}(r-1)}
$$

where $r=(3-2 F)^{1 / 2}$. Thus the generating function $F(x)=\sum k^{2} n_{k} e^{k x}$ $=\sum k^{2} n_{k} z^{k}$ is determined implicitly. The $n_{k}$ follow by expanding $F$ about $z=0$, and $n_{1}$ is determined by

$$
n_{1}=\lim _{z \rightarrow 0} F / z=6(2-\sqrt{3}) e^{\sqrt{3}-3} \simeq 0.452
$$

The higher $n_{k}$ can be found from (4.5) using Lagrange's expansion ${ }^{(21)}$ for this function, given parametrically as $z=z(\tau)$ and $F=F(\tau)$, where

$$
\begin{aligned}
\tau & =\sqrt{3}(\sqrt{3}-r) \\
F & =\tau(1-\tau / 6) \\
z^{-1} & =n_{1}(1-\tau / 6) \tau^{-1} e^{\tau}
\end{aligned}
$$

The result is

$$
F=\sum_{k=1}^{\infty} \frac{\left(n_{1} z\right)^{k}}{k !}\left[\left(\frac{d}{d \tau}\right)^{k-1}\left(1-\frac{\tau}{3}\right)\left(1-\frac{\tau}{6}\right)^{k} e^{\tau k}\right]_{\tau=0}
$$

and one finds after some algebra

$$
n_{k}=n_{1}^{k} \sum_{l=0}^{k-1} \frac{(k+l+1)(k-1) ! k^{k-3-l}}{l !(k-l+1) !(k-l-1) !}\left(-\frac{1}{6}\right)^{l}
$$

The first new terms read

$$
\begin{array}{cc}
n_{2}=n_{1}^{2} / 6, & n_{3}=n_{1}^{3} / 12 \\
n_{4}=n_{1}^{4} 7 / 108, & n_{5}=n_{1}^{5} 83 / 1296
\end{array}
$$

These can be found more simply from the recursion relation (3.9) with $s_{k}=k^{2}$.

The value of $b$ follows from (4.2) and (3.17), taking the limit $x \rightarrow 0$ : $b=\left[G^{\prime}(0)\right]^{-1}=\sqrt{3}$ since $G(0)=1 / 2$ [see (3.16)]. The most important quantities, in terms of the original variables follow from (3.8), (4.6), and (3.6) with $s_{k}=k^{2}$ as

$$
\begin{gathered}
c_{1}\left(t_{c}\right)=b n_{1}=6(2 \sqrt{3}-3) e^{\sqrt{3}-3} \\
b=M_{2}\left(t_{c}\right)=\sqrt{3}
\end{gathered}
$$


Thus we have determined all parameters (except $t_{c}$ ) in the postgel solution (3.3) for the model $s_{k}=k^{2}$, and obtain what constitutes a new solution to the kinetic equation (1.1). One may also determine the large $k$-behavior of $n_{k}$ from (4.9). However, it is much simpler to analyze (4.5) about $x=0$ to find the asymptotic behavior:

$$
c_{k}=\sqrt{3} n_{k}=3^{1 / 4}(2 \pi)^{-1 / 2}\left(k^{-7 / 2}+\frac{3 \sqrt{3}}{8} k^{-9 / 2}+\cdots\right)
$$

in agreement with (2.22) with $\dot{M}_{1}=-b$ and $\dot{M}_{2}=-b^{2}$, as follows from (3.20) and (4.11).

\subsection{The Case $s_{k}=k^{\omega}$}

Although in this more general case the equations for $F$ and $G$ resist solution, we can find $n_{1}$ and $b$ numerically, thus verifying that (3.3) gives a consistent solution. Furthermore, for large $\omega$ the solutions can be determined analytically.

The problem of determining $n_{1}$ from (3.9) can be taken care of as follows: Introduce $\tilde{n}_{k} \equiv n_{k} / n_{1}^{k}$. These $\tilde{n}_{k}$ satisfy an equation identical to (3.7), but with the boundary condition $\tilde{n}_{1}=1$. As follows from (3.19), the singularity in the generating function,

$$
h(z) \equiv F(\log z)=\sum k^{\omega} n_{k} z^{k} \simeq 1-b^{-1 / 2}(1-z)^{1 / 2}+\cdots
$$

located closest to the origin, is at $z=1$, so that the series only converges for $|z|<1$. Consequently, the radius of convergence of the series

$$
\tilde{h}(z)=\sum k^{\omega} \tilde{n}_{k} z^{k}=h\left(z / n_{1}\right)
$$

equals $n_{1}$, and can be determined numerically from the ratio test:

$$
n_{1}=\lim _{k \rightarrow \infty} \tilde{n}_{k-1} / \tilde{n}_{k}
$$

The nature of the singularity in $h(z)$ is known from (4.13)-(4.14) and implies that $n_{k}$ should have the asymptotic behavior

$$
\tilde{n}_{k} \simeq\left(n_{1}\right)^{-k} C k^{-\omega-3 / 2}
$$

on account of (2.9) with $C=(2 \pi b)^{-1 / 2}$ [see (3.20)]. Once $n_{1}$ is known, $b$ follows simply from (3.11). The results of this numerical procedure are listed in Table I. We also determined the constant $C$ in (4.16) and found agreement with $C=(2 \pi b)^{-1 / 2}$.

The solution of the recursion relation for large $\omega$ can be obtained analytically. Observe that $k^{\omega} \gg 1$ for all $k \geqslant 2$, so that (3.9) in lowest 
Table I. Values of $c_{1}\left(t_{c}\right)$ and $b=M_{\omega}\left(t_{c}\right)=2 M_{0}\left(t_{c}\right)$

for the Postgelation Solution (3.3)

in the Model $K_{i j}=(i j)^{\omega}$ as a Function of $\omega$

\begin{tabular}{rll}
\hline$\omega$ & \multicolumn{1}{c}{$c_{1}\left(t_{c}\right)$} & \multicolumn{1}{c}{$b$} \\
\hline 0.6 & 0.0344 & 0.1207 \\
0.7 & 0.1187 & 0.3818 \\
0.8 & 0.2076 & 0.6239 \\
0.9 & 0.2915 & 0.8290 \\
1.0 & $0.3679(=1 / e)$ & 1.0000 \\
1.1 & 0.4362 & 1.1419 \\
1.2 & 0.4967 & 1.2598 \\
1.5 & 0.6385 & 1.5100 \\
2.0 & 0.7836 & $1.7321(=\sqrt{3})$ \\
3.0 & 0.9132 & 1.9025 \\
4.0 & 0.9618 & 1.9591 \\
5.0 & 0.9823 & 1.9815 \\
10.0 & 0.9995 & 1.9995 \\
\hline
\end{tabular}

approximation may be written as

$$
k^{\omega} n_{k}=\frac{1}{2} \sum_{i+j=k}(i j)^{\omega} n_{i} n_{j} \quad(k \geqslant 2)
$$

This recursion relation must be solved under the restriction (3.10) or (3.12), valid to relative order $2^{-\omega}$. The generating function $F(x)$ satisfies

$$
F(x) \simeq 1-\left(1-2 n_{1} e^{x}\right)^{1 / 2}
$$

where $F(0)=1$ implies $n_{1}=1 / 2$. With the help of the binomial formula one finds

$$
n_{k} \simeq-\left(\begin{array}{c}
1 / 2 \\
k
\end{array}\right)(-1)^{k} k^{-\omega}=-\frac{(-1 / 2)_{k}}{k ! k^{\omega}}\left[1+O\left(2^{-\omega}\right)\right]
$$

where the Pochhammer symbol is defined as $(a)_{k}=\Gamma(k+a) / \Gamma(a)=$ $a(a+1) \ldots(a+k-1)$. The moments of $n_{k}$ are in the same approximation given by

$$
\sum k^{l} n_{k} \simeq \frac{1}{2}\left[1+O\left(2^{-\omega}\right)\right] \quad(l \ll \omega)
$$

so that $b=\left(\sum k n_{k}\right)^{-1}=2$. In the next approximation we neglect terms of relative order $3^{-\omega}$, i.e., (4.17) holds for $k \geqslant 3$ and the term with $k=2$ is obtained from the exact equation (3.9). The equation (4.18) for the generating function is replaced by

$$
F(x) \simeq 1-\left(1-2 n_{1} e^{x}-2 n_{2} e^{2 x}\right)^{1 / 2}
$$


where $F(0)=1$ implies $n_{1}+n_{2}=1 / 2$. Combination of the latter relation with (3.9) for $k=2$ yields

$$
\begin{aligned}
& n_{1} \simeq 1 / 2-2^{-\omega-3} \\
& n_{2} \simeq 2^{-\omega-3}+2^{-2 \omega-4}
\end{aligned}
$$

Then (4.21) takes the form

$$
F(x) \simeq 1-\left\{1-e^{x}\left[1-2^{-\omega-2}\left(1-e^{x}\right)\right]\right\}^{1 / 2}
$$

and one derives with the help of the binomial expansion $(k \rightarrow \infty)$ :

$$
k^{\omega} n_{k} \simeq-(-1 / 2)_{k} / k !+\frac{1}{4} 2^{-\omega}(-3 / 2)_{k} /[3(k-1) !]
$$

In the same approximation the moments for $l \ll \omega$ are given by

$$
\sum k^{l} n_{k} \simeq n_{1}+2^{l} n_{2} \simeq \frac{1}{2}-\frac{1}{8}\left(2^{l}-1\right) 2^{-\omega}
$$

so that

$$
b=\left(\sum k n_{k}\right)^{-1} \simeq 2-2^{-\omega-1}
$$

It is straightforward to include higher-order terms. We only mention the result for $n_{1}$ and $b$ up to relative order $4^{-\omega}$ :

$$
\begin{aligned}
n_{1} & \simeq 1 / 2-\left(2^{-\omega}+3^{-\omega}\right) / 8 \\
b & \simeq 2-2^{-\omega-1}-3^{-\omega} / 2
\end{aligned}
$$

Already the asymptotic results (4.22) and (4.26) are in good agreement with the numerical results in Table I for $\omega \geqslant 2$.

\subsection{The Case $s_{k}=e^{\alpha(k-1)}$}

For $\alpha>0$ the postgelation solution can be constructed in more or less explicit form. If $\alpha \leqslant 0$ the model does not show a gelation transition; if $0<\alpha \ll 1$ the kernel is almost unity for not too large values of $i$ and $j$, and the time evolution will be similar to that for the model $K_{i j}=1$. However, after some time the size distribution will "feel" the large $(i, j)$ behavior and the system will start to gelate. The gelation time is an unknown decreasing function of $\alpha$.

For a discussion of the generating function $F(x)$ of the special solution (3.3) it is most convenient to use the integral equation (3.18), where $\sigma(y)$ in (2.11) is given by $e^{\alpha} \delta(y-\alpha)$. It takes the form of a difference equation:

$$
F(x)-\frac{1}{2} F^{2}(x)=e^{\alpha} F(x-\alpha)
$$


to be solved, subject to the condition (3.16):

$$
F(0)=1
$$

The quantities $n_{1}, b$ and higher moments can be determined from (3.14), (3.15), and (3.17) as

$$
\begin{gathered}
\lim _{x \rightarrow-\infty} e^{-x} F(x)=n_{1} \\
1 / b=e^{\alpha} F^{\prime}(-\alpha) \\
\sum k^{l} n_{k}=e^{\alpha} F^{(l)}(-\alpha)
\end{gathered}
$$

where $F^{(l)}(x)$ denotes the $l$ th derivative. The method of solution goes as follows: Consider (4.28) as a recursion relation for $F(x)$, starting at $F(0)$ and yielding $e^{-x} F(x)$ at $x=-\infty$. Therefore also $e^{-x} F^{\prime}(x)$ is known at $x=-\infty$. Next, the first derivative of (4.28) is considered as a recursion relation for $F^{\prime}(x)$ to go into the reversed direction, and yielding $F^{\prime}(-\alpha)$ or $b$. A similar procedure can be followed to determine the moments $F^{(l)}(-\alpha)$.

As to the details of the procedure: we define a set of numbers

$$
p_{k}=e^{k \alpha} F(-k \alpha)
$$

which can be determined from the recursion relation

$$
p_{k+1}=p_{k}\left(1-\frac{1}{2} e^{-k \alpha} p_{k}\right) \quad\left(p_{0}=1\right)
$$

The limiting value $p_{\infty}$ gives

$$
p_{\infty}=\lim _{k \rightarrow \infty} p_{k}=n_{1}
$$

For $\alpha>0$ the $p_{k}$ constitutes a monotonically decreasing sequence of positive numbers, implying the existence of this limit (4.33). For $\alpha<0$ this limit does not exist, precluding the existence of the special postgelation solution. For $\alpha=0$ the $p_{k}$ 's in (4.32) approach the fixed point $p_{\infty}=0$, implying $n_{1}=0$, which also rules out the existence of the special post gelation solution.

If $\alpha$ is not too small, the analytic solution of (4.32) gives

$$
p_{\infty} \simeq \frac{1}{2}\left\{1-\frac{1}{4} e^{-\alpha}-\frac{1}{4} e^{-2 \alpha}-\frac{1}{8} e^{-3 \alpha}\right\}+O\left(e^{-4 \alpha}\right)
$$

and the $n_{k}$ can be determined as a power series in $e^{-\alpha}$, using (4.32) and the recursion relation (3.9). To obtain $b$ we introduce another set of numbers

$$
q_{k}=e^{k \alpha} F^{\prime}(-k \alpha)
$$

where $q_{\infty}=p_{\infty}=n_{1}$ on account of (3.14) and (4.33). A recursion relation 
can be obtained by differentiating (4.28)

$$
q_{k+1}=q_{k}\left(1-e^{-k \alpha} p_{k}\right) \quad\left(q_{\infty}=n_{1}\right)
$$

and the parameter $b$ in (4.30) is found as

$$
b=\left(q_{1}\right)^{-1}=\left(n_{1}\right)^{-1} \prod_{k=1}^{\infty}\left(1-e^{-k \alpha} p_{k}\right)
$$

where $b n_{1}=c_{1}\left(t_{c}\right)$. By taking the logarithm one shows that a nonvanishing limit exists for $\alpha>0$, thereby demonstrating the existence of the special postgel solution. Furthermore, the existence of $\sum e^{k \alpha} n_{k}$ in (3.6) guarantees that all moments $\sum k^{l} c_{k}\left(t_{c}\right)$ exist, and their calculation goes along similar lines. For instance, the second moment in (4.30) may be calculated from the recursion relation

$$
r_{k+1}=r_{k}\left(1-e^{-k \alpha} p_{k}\right)-e^{-k \alpha} q_{k}^{2} \quad\left(r_{\infty}=p_{\infty}\right)
$$

where $r_{k}$ is defined as

$$
r_{k}=e^{k \alpha} F^{\prime \prime}(-k \alpha)
$$

In general, the recursion relations must be solved numerically, yielding $b$, $n_{1}$, etc. for a given value of $\alpha$. For instance, if $\alpha=1$, one finds $b=$ 1.7103 and $c_{1}\left(t_{c}\right)=b n_{1}=0.7397$. In Table II $b$ and $c_{1}\left(t_{c}\right)$ are given as a function of $\alpha$.

Table II. Values of $c_{1}\left(t_{c}\right)$ and

$$
b=S_{0}\left(t_{c}\right)=2 M_{0}\left(t_{c}\right)
$$

for the Postgelation Solution (3.3)

in the Model $K_{i j}=\exp [\alpha(i+j-2)]$

as Function of $\alpha$

\begin{tabular}{ccc}
\hline$\alpha$ & $c_{1}\left(t_{c}\right)$ & $b$ \\
\hline 0.1 & 0.0650 & 0.4657 \\
0.2 & 0.1765 & 0.7924 \\
0.3 & 0.2877 & 1.0302 \\
0.4 & 0.3870 & 1.2086 \\
0.5 & 0.4724 & 1.3455 \\
1.0 & 0.7397 & 1.7103 \\
1.5 & 0.8618 & 1.8533 \\
2.0 & 0.9228 & 1.9201 \\
3.0 & 0.9739 & 1.9735 \\
4.0 & 0.9907 & 1.9906 \\
5.0 & 0.9966 & 1.9966 \\
\hline
\end{tabular}


We note for completeness that the small- $x$ behavior of $F(x)$ can be obtained from

$$
F(x)=1-\left[1-2 e^{\alpha} F(x-\alpha)\right]^{1 / 2}
$$

by expanding $F(x-\alpha)$ around $-\alpha$, where all derivatives $F^{(l)}(-\alpha)$ exist. The large- $k$ behavior of $n_{k}$ [or $c_{k}\left(t_{c}\right)$ ] is then found as

$$
n_{k} \simeq(2 \pi b)^{-1 / 2} k^{-3 / 2} e^{-(k-1) \alpha}\left\{1+(3 / 8) M_{2}\left(t_{c}\right) k^{-1}+\cdots\right\}
$$

The limiting case $\alpha \gg 1$ is completely analogous to the case of large $\omega$, studied in the previous subsection. In (4.40) we may introduce the approximation

$$
e^{\alpha} F(x-\alpha) \simeq n_{1} e^{x}+n_{2} e^{2 x}+\cdots
$$

so that $F(x)$ is again given by (4.21). The results, corresponding to (4.24) and (4.25), are

$$
e^{(k-1) \alpha} n_{k} \simeq-(-1 / 2)_{k} / k !+\frac{1}{4}(-3 / 2)_{k} e^{-\alpha} /[3(k-1) !]
$$

and

$$
\sum k^{l} n_{k} \simeq \frac{1}{2}+\frac{1}{8}\left(2^{l}-1\right) e^{-\alpha}
$$

so that

$$
b=\left(\sum k n_{k}\right)^{-1} \simeq 2-\frac{1}{2} e^{-\alpha}
$$

Higher-order terms may be obtained in a straightforward manner. First the limiting case $0<\alpha \ll 1$ the difference equation (4.28) may be approximated by the differential equation

$$
F^{\prime} \simeq F+(1 / 2 \alpha) F^{2} \quad[F(0)=1]
$$

where only the leading $\alpha$-correction is kept. The solution will give the correct $n_{k}$ for not too large values of $k$, i.e., $\alpha k \ll 1$, and reads to dominant order in $\alpha$

$$
F(x) \simeq 2 \alpha e^{x} /\left(2 \alpha+1-e^{x}\right)
$$

from which $b \simeq \frac{1}{2} \alpha$ follows on account of (4.30), and

$$
c_{k}\left(t_{c}\right)=b n_{k} \simeq \alpha^{2} /(2 \alpha+1)^{k} \quad(\alpha k \ll 1)
$$

\section{PREgelation STAGE}

In previous sections the main emphasis has been on the $k$ dependence of the size distribution in the postgelation stage. In this section we consider the time dependence of $c_{k}(t)$ in the pregelation stage. 


\subsection{Gelation Criteria from Moment Equations}

For the coagulation equation one is in general interested in such questions as: what are the characteristic kernels that cause gelation; what are the types of singularities involved before and after the gel point (critical exponents); how does the gelation time depend on the characteristics of the model and of the initial distribution, etc.?

In two previous sections these questions have been considered for the postgelation stage. In the pregelation stage the moment equations furnish a convenient tool to investigate such questions. They enable us to derive bounds on $t_{c}$ and values for the critical exponents, as we shall see later on (Section 5.2). We also argue that the solution $c_{k}(t)$ has only one singular point $t_{c}$, such that the moment equations remain valid up to that point. All these arguments are with the usual proviso that initial solutions exist for the kernels considered.

We start with the moment equations. Following White ${ }^{(14)}$ we introduce the partial moments

$$
M_{\alpha, L}(t)=\sum_{i=1}^{L} i^{\alpha} c_{i}(t)
$$

They satisfy the equations

$$
\dot{M}_{\alpha, L}=\frac{1}{2} \sum_{i=1}^{L-1} \sum_{j=1}^{L-i} c_{i} c_{j} K_{i j}\left\{(i+j)^{\alpha}-i^{\alpha}-j^{\alpha}\right\}-\sum_{i=1}^{L} \sum_{j=L-i+1}^{\infty} c_{i} c_{j} K_{i j} i^{\alpha}
$$

as can be derived from the kinetic equation (1.1) by multiplying with $k^{n}$, summing and rearranging terms. If

$$
\lim _{L \rightarrow \infty} \sum_{i=1}^{L} c_{i} K_{i j} i^{\alpha}<\infty
$$

for all $j$, then the second term on the right-hand side of (5.2) gives a vanishing contribution in the limit of large $L$, and one obtains the equations $^{(2)}$

$$
\dot{M}_{\alpha}=\frac{1}{2} \sum_{i=1}^{\infty} \sum_{j=1}^{\infty} c_{i} c_{j} K_{i j}\left[(i+j)^{\alpha}-i^{\alpha}-j^{\alpha}\right]
$$

For integral $\alpha$ values and for kernels of the form $K_{i j}=s_{i} s_{j}$ they lead to the moment equations:

$$
\begin{aligned}
& \dot{M}_{0}=-\frac{1}{2} S_{0}^{2} \\
& \dot{M}_{n}=\frac{1}{2} \sum_{l=1}^{n-1}\left(\begin{array}{c}
n \\
l
\end{array}\right) S_{l} S_{n-l} \quad(n \geqslant 2)
\end{aligned}
$$


where

$$
S_{n}(t)=\sum_{k=1}^{\infty} k^{n} s_{k} c_{k}(t)
$$

For $s_{k}=k^{\omega}$ we have in particular $S_{n}=M_{n+\omega}$. The equations for $\dot{M}_{n}$ are only valid as long as $S_{n}$ is finite [see (5.3)].

At this point we can see how the gelation transition occurs. Gelation means that at a specific time mass conservation is violated, i.e., $\lim _{L \rightarrow \infty} \dot{M}_{1, L} \neq 0$. This happens when $S_{1}$ diverges, as follows from $(5.2)$ and (5.3). As an illustration we consider the classical case, $s_{k}=k(\omega=1)$ where the gel point is determined by the divergence of $S_{1}(t)=M_{2}(t)$. The equation for $M_{2}(t)$ in (5.5) reads in this case $\dot{M}_{2}=M_{2}^{2}$ and has the solution

$$
M_{2}(t)=\left[M_{2}^{-1}(0)-t\right]^{-1}
$$

Hence for any initial distribution the gel point is given by $t_{c}=1 / M_{2}(0)$, and the exponent $\gamma$ in (1.6) equals unity.

There is also an indication of the occurrence of gelation in the behavior of $M_{0}(t)$. Consider again the classical case, $\omega=1$, where (5.5) for $n=0$ has the solution $\left[S_{0}(t)=M_{1}(t)=1\right.$ before gelation]:

$$
M_{0}(t)=M_{0}(0)-\frac{1}{2} t
$$

This $M_{0}(t)$ vanishes at $t_{0}=2 M_{0}(0)$, which is larger than $t_{c}=1 / M_{2}(0)$ for all $c_{k}(0)$. It does not mean that the total number of clusters vanishes within a finite time $t_{0}$, but the moment equation with $M_{1}(t)=1$ is no longer valid ${ }^{6}$ for $t \geqslant t_{c}$. In general, ${ }^{(8)}$ the vanishing of $M_{0}(t)$ within a finite time $t_{0}$ is an indication that gelation occurs at an earlier point $t_{c}$.

For more general models one cannot solve the moment equations explicitly, but one can still use them to obtain bounds on the moments, providing criteria for the occurrence of gelation. Before doing so we want to argue that the solution $\left[c_{k}(t)\right]$ has only one singular point $\left(t=t_{c}\right)$.

McLeod $^{(13)}$ has shown that initial solutions of the coagulation equation with $K_{i j} \leqslant C i j(i, j \rightarrow \infty)$ are bounded for $t>1 / e$ by the exponentially decaying solutions (3.1) of the classical case with $K_{i j}=C i j$, so that all moments $M_{n}$ exist on this time interval. Leyvraz and Tschudi ${ }^{(4)}$ argue that there is no reason to imagine the occurrence of a singularity before $t_{c}$, that would change the exponential decay of $c_{k}(t)$ without causing gelation, and therefore assume that the exponential decay persists up to $t_{c}$.

As additional support for this assumption we show using the same heuristic method as in Section 2.1, that no solutions of (1.1) exist that

\footnotetext{
${ }^{6}$ For monodisperse initial conditions $M_{0}(t)=M_{0}\left(t_{c}\right) / t$ for $t \geqslant t_{c}$ by virtue of (3.2).
} 
conserve mass and have an algebraic tail $c_{k}(t) \sim k^{-\alpha-1}$ with nonintegral ${ }^{7}$ $\alpha>1$. For definiteness we take the kernel $K_{i j}=s_{i} s_{j}$ with $s_{k}=k^{\omega}$ and $\omega>1 / 2$. The assumed algebraic tail in $c_{k}$ corresponds to small- $x$ singularities: $(-x)^{\alpha}$ in $g(x, t)$ and $(-x)^{\alpha-\omega}$ in $f(x, t)$ on account of (A.4) and (2.2). Hence all $M_{n}$ with $n>[\alpha]$ diverge. The assumed mass conservation implies $S_{1}=M_{1+\omega}<\infty$ on account of (5.3). Then the generating functions have the small- $x$ behavior

$$
\begin{aligned}
& f(x, t)=S_{0}+x S_{1}+\cdots+o\left(x^{\alpha-\omega}\right)+\cdots \\
& g(x, t)=M_{0}+x M_{1}+\cdots+O\left(x^{\alpha}\right)+\cdots
\end{aligned}
$$

Inserting (5.9) into (2.7) with $\dot{M}_{1}=0$ (no gelation) shows that no $\alpha$ can be found, such that the equation is satisfied.

Therefore, solutions with an algebraic tail, but without gelation do not exist, and the only singularity in $c_{k}(t)$ occurs at $t_{c}$. This implies that $M_{n}(t)<\infty$ and $S_{n}(t)<\infty$ for all $n$ and $t<t_{c}$, and that $M_{n}\left(t_{c}\right)=\infty$ for $n \geqslant 1+\omega$ and $S_{n}\left(t_{c}\right)=\infty$ for $n \geqslant 1$. Thus, whenever some moment diverges, gelation occurs at that point. We assume that the same conclusions apply for all kernels $K_{i j}$, for which the kinetic equation is well defined.

These heuristic arguments are only positive evidence, but not a proof for the absence of more singular points. ${ }^{8}$

We return to bounds on moments, which will be used to test if gelation will or will not occur for a given coagulation kernel. The divergence of a lower bound of some moment $M_{n}(n>1)$ is an indication that the gelation point has been reached. An upper bound on $M_{n}(n>1)$ remaining finite for all $t \geqslant 0$ excludes gelation. Similarly, upper bounds for $M_{0}$, vanishing within a finite time $t_{0}$, indicate that gelation occurs before $t_{0}$. The bounds on moments also provide bounds on $t_{c}$.

A useful inequality for our purpose is Jensen's inequality, ${ }^{(22)}$ which can be formulated as follows: given a convex function $\psi(x)$ and a set of positive numbers $a_{k}$ from which for any set $b_{k}$ expectation values are calculated as

$$
E(b)=\sum b_{k} a_{k} / \sum a_{k}
$$

\footnotetext{
${ }^{7}$ The arguments for integral $\alpha$ require minor modifications (see Appendix A) and lead to the same conclusions.

${ }^{8}$ Suppose that the set $\left\{c_{k}(t)\right\}$ would have more singular points $t_{i}$ (with $i=1,2, \ldots$ and $\ldots t_{2}<t_{1}<t_{c}$ ) with algebraic decay $c_{k} \sim k^{-1-t_{i}}$ in the interval $t_{i} \leqslant t_{c}<t_{i-1}$, then the moments $M_{l}(t)$ with $l \geqslant l_{i}$ diverge in this interval [for definiteness, take $K_{i j}=(i j)^{\omega}$, so that the corresponding $S_{l-\omega}(t)$ would also diverge], and the equation for $\dot{M}_{n}$ with $n=l_{i}-\omega$ would only be valid for $t<t_{i}$, since $S_{n}\left(t_{i}\right)=M_{l_{i}}\left(t_{i}\right) \rightarrow \infty$. Consequently (conclusions obtained from) the moment equations would only be valid for $t$ smaller than the minimum of $t_{i}$, which could be zero.
} 
the following inequality holds:

$$
E(\psi(b)) \geqslant \psi(E(b))
$$

The inequality is strict unless $\psi(x)=x$. For concave functions the inequality is reversed. All required sums must converge. We shall further employ inequalities like

$$
\begin{gathered}
M_{\alpha+\beta} \geqslant M_{\alpha} \\
\sum_{i, j}\left(i^{\gamma}-j^{\gamma}\right)^{2} i^{\alpha} j{ }^{\beta} c_{i} c_{j}=M_{\alpha+2 \gamma} M_{\beta}+M_{\beta+2 \gamma} M_{\alpha}-2 M_{\alpha+\gamma} M_{\beta+\gamma} \geqslant 0 \\
\text { for } \alpha, \beta, \gamma>0 .
\end{gathered}
$$

As a simple application for the models with $s_{k}<k(k \rightarrow \infty)$ we have from (5.5) for $n=2$

$$
\dot{M}_{2}=S_{1}^{2}<M_{2}^{2}
$$

implying that the right-hand side of (5.7) represents an upper bound on $M_{2}(t)$. A possible gel point must then necessarily lie beyond the time $1 / M_{2}(0)$. Stronger inequalities are needed to exclude or prove gelation. For the case $s_{k}>k(k \rightarrow \infty)$ the inequality (5.12) is reversed showing that gelation occurs and that the gel point must lie before $1 / M_{2}(0)$. Physically this is expected as larger coagulation rates are likely to shift the gel-point to shorter times.

In the following application we consider a class of coagulation kernels

$$
K_{i j}=\psi(i, j)
$$

where $\psi(i, j)$ is a positive homogeneous function of degree $\lambda$, i.e.,

$$
\psi(s i, s j)=s^{\lambda} \psi(i, j)
$$

that is either a convex or concave function of both variables $i$ and $j$. Homogeneous kernels very frequently appear in coagulation problems. ${ }^{(2,12,23)}$ Concerning gelation we shall derive the following criteria ${ }^{9}$ for homogeneous kernels:

(i) If $\psi(i, j)$ is convex and $\lambda>1$ gelation occurs;

(ii) If $\psi(i, j)$ is concave and $\lambda \leqslant 1$ no gelation occurs.

To prove this we take (5.4) for $\alpha=0$ and 2:

$$
\begin{aligned}
& \dot{M}_{0}=-\frac{1}{2} \sum_{i, j} c_{i} c_{j} \psi(i, j) \\
& \dot{M}_{2}=\sum_{i, j} c_{i} c_{j} \psi(i, j) i j
\end{aligned}
$$

${ }^{9}$ Provided, of course, that initial solutions exist and that kinetic equations are well-defined for all $t \geqslant 0$. 
We first take $\psi(i, j)$ convex and apply Jensen's inequality (5.10) twice in (5.14b) with $a_{k}=k c_{k}, b_{k}=k$, and $E(k)=M_{2} / M_{1}=M_{2}$ (before gelation). This yields the inequality

$$
\dot{M}_{2}>M_{1}^{2} \psi\left(\frac{M_{2}}{M_{1}}, \frac{M_{2}}{M_{1}}\right)=\psi(1,1) M_{2}^{\lambda}
$$

After integration we obtain the lower bounds:

$$
\begin{array}{ll}
M_{2}(t)>M_{2}(0)\left(1-t / t_{2}\right)^{-1(\lambda-1)} & (\lambda>1) \\
M_{2}(t)>M_{2}(0) \exp [\psi(1,1) t] & (\lambda=1) \\
M_{2}(t)>M_{2}(0)\left(1+t / t_{2}\right)^{1 /(1-\lambda)} & (\lambda<1)
\end{array}
$$

with

$$
t_{2}=\left[\psi(1,1)|\lambda-1| M_{2}^{\lambda-1}(0)\right]^{-1}
$$

Similarly we derive for $M_{0}$ the upper bounds

$$
\begin{array}{ll}
M_{0}(t)<M_{0}(0)\left(1-t / t_{0}\right)^{1 /(\lambda-1)} & (\lambda>1) \\
M_{0}(t)<M_{0}(0) \exp \left[-\frac{1}{2} \psi(1,1) t\right] & (\lambda=1) \\
M_{0}(t)<M_{0}(0)\left(1+t / t_{0}\right)^{-1 /(1-\lambda)} & (\lambda<1)
\end{array}
$$

where

$$
t_{0}=2[\psi(1,1)|\lambda-1|]^{-1}\left[M_{0}(0)\right]^{\lambda-1}
$$

The inequalities for $\lambda>1$ prove criterion (i) for convex $\psi(i, j)$, and provide an upper bound for the gel point, i.e.,

$$
t_{c} \leqslant t_{2}
$$

One easily verifies that $t_{0} \geqslant 2 t_{2}>0$ for all possible initial distributions when $\lambda>1$. For $\lambda \leqslant 1$ all lower bounds remain finite and nonvanishing on finite time intervals, and gelation is not excluded. For concave $\psi(i, j)$ all inequalities in (5.16) and (5.18) are reversed. The resulting inequalities for $\lambda \leqslant 1$ prove criterion (ii), since the limit (5.3) exists for $\alpha=1$, as $\sum_{i} c_{i} \psi(i, j) i$ $<C_{j} M_{2}<\infty$. In the case $\lambda>1$ and concave $\psi$ gelation may or may not occur. If it does, $t_{c} \geqslant t_{2}$.

The above criteria, applied to the kernel $K_{i j}=(i j)^{\omega}$ (where $\lambda=2 \omega$ ), yield gelation for $\omega>1$ (the convex case), and exclude gelation for $\omega$ $\leqslant 1 / 2$. For $\omega>1$ it yields an upper bound for $t_{c}$ :

$$
t_{c} \leqslant\left\{(2 \omega-1)\left[M_{2}(0)\right]^{2 \omega-1}\right\}^{-1}
$$


For $\omega<1$ the inequality (5.21) is reversed and a lower bound results. The bound reaches its largest value, $(2 \omega-1)^{-1}$, for monodisperse initial conditions, $c_{k}(0)=\delta_{k 1}$.

We conclude this subsection by deriving a stronger inequality for the special case $K_{i j}=s_{i} s_{j}$ with $s_{k}=k^{\omega}$. This will provide a better bound on $t_{c}$ for all $\omega>1 / 2$. We take the moment equation (5.4) and substitute $\alpha=2 \omega$ :

$$
\dot{M}_{2 \omega}=\frac{1}{2} \sum_{i, j}(i j)^{2 \omega} c_{i} c_{j} x(i, j)
$$

where we introduce the function

$$
\chi(i, j) \equiv\left[(i+j)^{2 \omega}-i^{2 \omega}-j^{2 \omega}\right] /(i j)^{\omega}
$$

which is homogeneous of degree zero $(\lambda=0)$. At fixed $j$ and $\omega>1$ it reaches a minimum for $i=j$, which is independent of $j$. Thus we have $\chi(i, j) \geqslant \chi(j, j)=2^{2 \omega}-2$, and hence from (5.22)

$$
\dot{M}_{2 \omega} \geqslant\left(2^{2 \omega-1}-1\right) M_{2 \omega}^{2}
$$

Integration of this equation yields a lower bound on $M_{2 \omega}(t)$ :

$$
M_{2 \omega}(t) \geqslant M_{2 \omega}(0)\left(1-t / t_{0}\right)^{-1}
$$

with

$$
t_{0}=\left[\left(2^{2 \omega-1}-1\right) M_{2 \omega}(0)\right]^{-1}
$$

In the (physical) range $1 / 2<\omega \leqslant 1$ the inequality (5.24) is reversed. In summary

$$
\begin{array}{ll}
t_{c} \leqslant t_{0} & \text { for } \quad \omega>1 \\
t_{c} \geqslant t_{0} & \text { for } \quad 1 / 2<\omega \leqslant 1
\end{array}
$$

\subsection{Scaling Postulate and Similarity Transformations}

In this subsection we investigate scaling and its consequence in the pregelation stage. For the model $K_{i j}=(i j)^{\omega}$ with $1 / 2<\omega \leqslant 1$ we obtain some additional support for the exponent relations of Leyvraz and Tschudi, using moment equations and similarity transformations. We further show that scaling cannot be true for general coagulation kernels. Finally we derive a scaling relation for the gelation time for a class of initial distributions.

If scaling holds for $t \uparrow t_{c}$, some additional information on the exponent $\tau$ and $\sigma$ can be obtained by inserting the scaling prediction (1.10) into the 
moment equations (5.5). By requiring these equations to be satisfied to dominant order in $\left(t_{c}-t\right)$ we find the exponent relation

$$
\sigma+\tau=2 \omega+1
$$

[in agreement with (1.4) and (1.12)], and a set of relations for $B_{n}$. If one assumes in addition that $\tau$ is the same before and after gelation (i.e., $\tau=\omega+3 / 2$ ) - this is supported by the results of Leyvraz and Tschudi ${ }^{(4)}$ then the explicit predictions ${ }^{(4)}(1.12)$ and (1.13) for $\sigma$ and $\gamma$ are obtained. In some cases the relations for the (positive) coefficients $B_{n}$ provide a further test for the validity of scaling. This happens for the kernels $K_{i j}=(i j)^{\omega}$ and integral $\omega$-values, where the moment equations (5.5) are closed, as $S_{n}$ $=M_{n+\omega}$. Consequently, the coefficients $B_{n}$ satisfy a set of recursion relations, whose solutions must be positive. In the classical case, $\omega=1$, where scaling is known to be valid, the moments have the form (1.10), i.e., $M_{n}(t) \simeq B_{n}\left(t_{c}-t\right)^{-2 n+3}$ as $t \uparrow t_{c}$, and the solution ${ }^{10}$ of the recursion relations yield positive results for $B_{n}$.

Next, we consider the case, $\omega=2$, where $\sigma=3 / 2$. The dominant part of the moments as $t \uparrow t_{c}$ is according to (1.10)

$$
M_{n}(t) \simeq B_{n}\left(t_{c}-t\right)^{(5-2 n) / 3}
$$

where the coefficients satisfy the recursion relation

$$
[(2 n-3) / 5] B_{n}=\frac{1}{2} \sum_{l=1}^{n-1}\left(\begin{array}{c}
n \\
l
\end{array}\right) B_{l+2} B_{n-l+2}
$$

with $n \geqslant 3$. Successive solution of (5.29) for $n=3,4 \ldots$ shows that $B_{7}$ becomes negative (unphysical). The conclusion is that the scaling postulates do not hold for $\omega=2$. However, the cases $s_{k}=k^{\omega}$ with $\omega=2$, and in general $\omega>1$ are already unphysical, in that the surface area grows faster than the volume $\sim k$.

Another example in which the scaling postulate (1.9) cannot be true ${ }^{11}$ is the model $K_{i j}=s_{i j}$ with $s_{k}=\exp [\alpha(k-1)]$, since $s_{k} c_{k}(t)$ must be finite at all times [to have a well-defined kinetic equation (1.1)] implying the same property for all $M_{n}(t)$.

The validity of the exponent relation (5.27) is further supported by invariance properties of the coagulation equation under similarity transformations. In view of the fundamental importance of scaling we investigate possible pregelation types of similarity solutions to the coagulation equa-

${ }^{10}$ The solution for $\omega=1$ is $B_{2}=1$ and $B_{n}=(2 n-5) ! ! B^{n-2}$ for $n>2$, where $(2 n+1) ! !=$ $1 \cdot 3 \cdot 5 \ldots(2 n+1)$, with $B$ undetermined, in agreement with the exact solution for $t \uparrow t_{c}$. ${ }^{11} \mathrm{~A}$ modified form of (1.9) might still hold as $t \uparrow t_{c}$ :

$$
c_{k}(t) \simeq c_{k}\left(t_{c}\right) \Phi\left(k\left(t_{c}-t\right)^{1 / \sigma}\right) \simeq s_{k}^{-1} k^{-\tau} \Phi\left(k\left(t_{c}-t\right)^{1 / \sigma}\right) .
$$


tion with homogeneous kernels (5.13) following Lushnikov, ${ }^{(11)}$ and Silk and White. ${ }^{(23)}$ Since the scaling property is formulated for large $k$ we may conveniently consider the continuous version of the coagulation equation with $K_{i j}=\psi(i, j)$

$$
\frac{\partial c(k, t)}{\partial t}=\frac{1}{2} \int_{0}^{k} d l \psi(l, k-l) c(l, t) c(k-l, t)-c(k, t) \int_{0}^{\infty} d l \psi(k, l) c(l, t)
$$

where the size distribution is considered as a continuous function of $k$. We observe that this equation is invariant under the group of similarity transformations (with $s>0$ ):

$$
\begin{aligned}
\bar{k} & =s k \\
\bar{t} & =s^{-\alpha} t \\
\bar{c}(\bar{k}, \bar{t}) & =s^{-\theta} c(k, t)
\end{aligned}
$$

provided the exponents $\sigma$ and $\theta$ are related as

$$
\sigma+\theta=\lambda+1
$$

It can be verified by direct substitution into (5.30). A similarity solution of (5.30) is itself invariant under the group of transformations (5.31), and must have the property ${ }^{(24,25)}$

$$
c(k, t)=s^{\theta} c\left(s k, s^{-\sigma} t\right)
$$

for all $s>0$. Furthermore (5.30) is invariant under time translations. Hence in the pregelation stage we may replace $t$ by the positive quantity $\left(t_{c}-t\right)$, so that possible similarity solutions will have the form [choose $s=k^{-1}$ in (5.32)]:

$$
c(k, t)=k^{-\theta} \Phi\left(k\left(t_{c}-t\right)^{1 / \sigma}\right)
$$

where $\sigma$ and $\theta$ satisfy (5.31b). For the kernels $\psi(i, j)=(i j)^{\omega}(\lambda=2 \omega)$ this is equivalent with (5.27). Substitution of (5.32) into (5.30) yields an equation for $\Phi(x)$. In fact a similarity solution is one in which the scaling property is valid for all times, not only near $t_{c}$.

An acceptable solution should satisfy the conditions of positivity and mass conservation. The latter condition imposes some further restrictions on $\theta$ and $\sigma$ (see below). We note, however that the solutions not satisfying mass conservation are of interest as we expect general solutions to approach a similarity form only close to (and below) the gel point (this is in fact the scaling postulate).

Indeed, the Flory-Stockmayer solution for $K_{i j}=i j$ is not itself a similarity solution, but only approaches for $t \uparrow t_{c}$ the similarity form given in 
footnote 5 with $\Theta=\tau=5 / 2$ and $\sigma=1 / 2$, in agreement with (5.3lb). Similarity solutions to the coagulation equation were studied by Lushni$\operatorname{kov}^{(11)}$ and Silk and White, ${ }^{(23)}$ who imposed mass conservation on $c(k, t)$. This implies the exponent relations

$$
\theta=2, \quad \sigma=\lambda-1
$$

and the similarity form ${ }^{12}$

$$
c(k, t)=k^{-2} \Phi\left(k t^{1 / \sigma}\right)
$$

However, the Flory-Stockmayer solution does not approach this form.

Given an initial solution $c(k, t)$ of the coagulation equation in the pregelation stage (it necessarily conserves mass), we can use the similarity transformation (5.31a) to construct a new solution $\bar{c}(k, t)$, the exponents of which are given by (5.34), i.e.,

$$
\bar{c}(k, t)=s^{2} c\left(s k, s^{1-\lambda} t\right)
$$

This corresponds to a transformation of the corresponding initial conditions and leads to a transformed value of the gelation time:

$$
t_{c}(s)=t_{c}(1) s^{\lambda-1}
$$

The gelation time is a function of the initial conditions [a combination of fractional powers of moments $\left.M_{n}(0)\right]$ that transforms according to (5.37). Since under (5.36) $M_{\lambda}^{-1}(0)$ transforms exactly as $t_{c}$ in (5.37), a possibility (among many others) could be

$$
t_{c} \simeq \frac{A(\lambda)}{M_{\lambda}(0)}
$$

Supposing, for the sake of argument, that (5.38) were exact (which is not the case), we could find $M_{\lambda}(t)$ by the following argument. By letting the system evolve from $t=0$ to $t$ and considering the size distribution at time $t$ as the new initial distribution (for which obviously $t_{c}^{\prime}=t_{c}-t$ ) we deduce from (5.38)

$$
M_{\lambda}(t) \simeq \frac{M_{\lambda}(0)}{1-t / t_{c}}
$$

which implies

$$
t_{c} \simeq \frac{\dot{M}_{\lambda}(0)}{M_{\lambda}(0)}=\frac{1}{2^{\lambda-1}-1}
$$

\footnotetext{
${ }^{12}$ Similarity solutions or self-preserving solutions with $\omega=0$ are of interest in aerosol coagulation, ${ }^{(2)}$ and in polymerization of linear chains. ${ }^{(5,6,8,26)}$
} 
for monodisperse initial conditions. For the kernel $K_{i j}=(i j)^{\omega}$ where $\lambda=2 \omega$ this value for $t_{c}$-which is not derived-equals the bound (5.25b). For $\omega=1$ all these values are exact. For $\omega=2$ we can verify that (5.39) with $\lambda=4$ is not an exact solution of the moment equation (5.5).

\subsection{Special Solution and Corresponding Initial Conditions}

In this section we consider the models for which we have found the special postgelation solution (3.3). The question $\operatorname{arises}^{(4)}$ whether (3.3) is reached from monodisperse initial conditions. The only case ${ }^{13}$ for which the answer is known is the case, $\omega=1$, where the solution (3.1) for $c_{k}(0)=\delta_{k 1}$ evolves into the special postgelation solution (3.2). One may speculate that the same is true for other models.

In order to decide this we construct some inequalities (under the usual assumptions that initial solutions exist and that the moment equations are valid up to $t_{c}$ ). We integrate (1.1) for $c_{1}(t)$ and (5.5) for $M_{0}$, using $c_{k}(0)=\delta_{k 1}$ with the result

$$
\begin{gathered}
-\log c_{1}\left(t_{c}\right)=\int_{0}^{t_{c}} d \tau S_{0}(\tau) \\
2-2 M_{0}\left(t_{c}\right)=\int_{0}^{t_{c}} d \tau S_{0}^{2}(\tau)
\end{gathered}
$$

If $S_{0}(t) \geqslant 1$ for $0 \leqslant t \leqslant t_{c}$, then

$$
-\log c_{1}\left(t_{c}\right) \leqslant 2-2 M_{0}\left(t_{c}\right)
$$

If $S_{0}(t) \leqslant 1$ for $0 \leqslant t \leqslant t_{c}$, the inequality is reversed.

Consider first the case $s_{k}=\exp \alpha(k-1)$. Since

$$
S_{0}(t)-1=\sum_{k}\left(e^{\alpha(k-1)}-k\right) c_{k}(t) \geqslant 0
$$

for $\alpha \geqslant \log 2$, the inequality (5.42) should be valid. However, one easily verifies with the help of Table II that the inequality does not hold for $\alpha \geqslant \log 2 \simeq 0.69$. For the remaining range of $\alpha$-values the question has not been decided.

We consider next the case, $\omega=2$, and assume that the monodisperse initial condition evolves into the special postgelation solution. Then $M_{2}(0)$ $=1$ and $M_{2}\left(t_{c}\right)=\sqrt{3}$ [see (4.11)], and it follows from the moment equations that $M_{2}(t)$ is a concave function. This implies

$$
M_{2}(t)<1+(\sqrt{3}-1) t / t_{c}
$$

Furthermore, since $S_{0}(t)=M_{2}(t)$ for the case $\omega=2$, we deduce from (5.41)

${ }^{13}$ More generally it is also true for $K_{i j}=[(f-2) i+2][(f-2) j+2]$ (see Ref. 9). 
by integration of (5.44) that

$$
t_{c}>-2 \log c_{1}\left(t_{c}\right) /(\sqrt{3}+1) \simeq 0.18
$$

where $c_{1}\left(t_{c}\right)$ is taken from (4.11). On the other hand, the inequality (5.24) requires that $t_{c}<1 / 7 \simeq 0.14$ for monodisperse initial conditions. 'These conflicting bounds invalidate our assumption for the case $\omega=2$. Using (5.4) and assuming that the special postgelation solution evolves from the monodisperse initial condition one finds the lower bound $\left[-\log c_{1}\left(t_{c}\right)\right] / b$. For all $\omega>1.1$ this exceeds the upper bound (5.26) (see Table I) and again the assumption cannot be true. In the physically interesting range $1 / 2<\omega$ $\leqslant 1$, where the existence of the postgelation solution has been proved, we have no further evidence but we expect that the special postgelation solution is only related to the monodisperse initial condition in the classical case $\omega=1$.

\subsection{Taylor Series Expansions for the Case $K_{i j}=(i j)^{\omega}$}

If one expands the size distribution $c_{k}(t)$ in a Taylor series, then the coefficients in this series can be found recursively as has been shown in Ref. 11 for general kernels. In the case of integral $\omega$-values the same is true for the moment equations. However, some problems arise, as we shall show. For the case, $\omega=2$, we obtain for monodisperse initial conditions

$$
M_{2}(t)=1+t+3 t^{2}+13 t^{3}+69 t^{4}+418.2 t^{5}+2789 t^{6}+20019 t^{7}+\cdots
$$

by calculating the $l$ th derivative of $M_{2}$ from the moment equations. Numerically the radius of convergence of this series seems to be zero (the same is true for higher moments), suggesting that $M_{2}(t)$ diverges at $t=0$, which would contradict the monodisperse initial condition, and would imply that solutions for the initial condition do not exist. Another possible explanation, however, is that (5.46) represents an asymptotic expansion of $M_{2}(t)$ about $t=0$. One may also try to obtain series solutions for the size distribution with monodisperse initial conditions. Following Lushnikov ${ }^{(1)}$ we insert the ansatz

$$
c_{k}(t)=\sum_{l=0}^{\infty} \gamma_{k l} t^{k+l-1}
$$

with $\gamma_{10}=1$ into the coagulation equation. This yields a set of recursion relations

$$
(k+l-1) \gamma_{k l}=\frac{1}{2} \sum_{i=1}^{k-1} \sum_{j=0}^{l} K_{i, k-i} \gamma_{i j} \gamma_{k-i, l-j}-\sum_{j=1}^{l} \sum_{m=0}^{l-j} K_{k j} \gamma_{k m} \gamma_{j, l-j-m}
$$


This set can be solved recursively along diagonals in the $(k, l)$-plane. For $K_{i j}=(i j)^{2}$ it is found again that the radius of convergence of any $c_{k}(t)$ considered approaches zero numerically. The numerical data for large $k$ and fixed (small) $l$ suggest that the leading coefficient can be represented by the relation

$$
\gamma_{k l} / \gamma_{k-1, l} \simeq k+1+2 l+a_{l} / k+\cdots
$$

where the $a_{l}$ for $l=0,1,2,3,4,5, \ldots$ are approximately given by $3,7,14$, $15,39,56, \ldots$, which suggests that $c_{k}(t)$ behaves at large $k$ as

$$
c_{k}(t) \simeq \gamma_{0}(k+1) ! t^{k-1}+\gamma_{1}(k+3) ! t^{k}+\cdots
$$

These numerical results are consistent with the bound $\gamma_{k 0} \geqslant[(k-1) !]^{\omega-1}$ valid for all $\omega$-values, that follows directly from the inequality

$$
(k-1) \gamma_{k 0} \geqslant K_{1, k-1} \gamma_{k-1,0}
$$

implied by the recursion relation (5.48) for $l=0$. Furthermore in the simple system, in which only monomer- $n$-mer interactions are allowed (a nucleation model with $K_{i j} \neq 0$ for $i$ and/or $j$ equal to unity) one can solve the recursion relation with the result

$$
\begin{aligned}
& \gamma_{k 0}=\frac{1}{2}[(k-1) !]^{\omega-1} \\
& \gamma_{k 1}=-\frac{1}{2}[(k-1) !]^{\omega-1} k^{-1} \sum_{l=1}^{k}\left(l^{\omega}+l-1\right)
\end{aligned}
$$

For the case, $\omega=2$, where

$$
\gamma_{k 0}=\frac{1}{2}(k-1) !, \quad \gamma_{k 1}=-(k-1) !\left(k^{2}+3 k-1\right) / 6
$$

the structure of the $t$-expansion is rather similar to (5.50).

Perhaps methods like Borel resummation can assign a meaning to these series, but the possibility remains that no pregelation solutions exist for the kernels $K_{i j}=(i j)^{\omega}$ with $\omega>1$.

\section{COAGULATION KERNELS OF THE FORM $\left(s_{i}+s_{j}\right)$ AND $\left(s_{i} r_{j}+s_{j} r_{i}\right)$}

In this section we are concerned with a new class of models. Our aim is to investigate for these models essentially the same questions, as posed in Sections 2 and 5 on the occurrence of gelation: does the kinetic equation admit postgelation solutions (with a time-dependent $M_{1}$ ), and if so what is the large- $k$ behavior of such solutions?

First we focus attention on kernels of the general form $s_{i}+s_{j}$. Existence of global solutions and absence of gelation has been rigorously proved by White ${ }^{(14)}$ for kernels $K_{i j} \leqslant C(i+j)(i, j \rightarrow \infty)$. The coagulation 
equation takes the form

$$
\dot{c}_{k}=\sum_{i+j=k} s_{i} c_{i} c_{j}-s_{k} c_{k} M_{0}-c_{k} S_{0}
$$

with $M_{0}$ and $S_{0}$ defined in (2.3) and (2.5). By using the generating functions $g$ and $f$, defined in (2.2), we obtain from (6.1)

$$
\dot{g}-\dot{M}_{0}=\left(f-S_{0}\right)\left(g-M_{0}\right)
$$

where the relation $\dot{M}_{0}=-M_{0} S_{0}$ has been used [take (5.4) with $\alpha=0$ ]. We want to investigate the possibility of a postgelation solution to (6.1). To this end we try a solution to (6.2) of the form (2.4) with $\dot{M}_{1}(t) \neq 0$. By taking the limit of small $x$ equation (6.2) reduces to the relation $x \dot{M}_{1}+\dot{\Delta}(x)$ $\simeq \delta(x)\left[x M_{1}+\Delta(x)\right]$. Its right-hand side vanishes as $o(x)(x \uparrow 0)$ and consistency requires $\dot{M}_{1}(t)=0$. Therefore, kernels $K_{i j}=s_{i}+s_{j}$ do not admit postgelation solutions with time-dependent mass $M_{1}$, irrespective of the functional form of $s_{k}$. However, this conclusion does not imply the existence of global solutions with $M_{1}(t)=1$ for the case $s_{k}>C k$, as will be argued for $s_{k}=k^{\omega}$ with $\omega>1$. According to (5.17), $M_{2}$ diverges at $t_{c}<\frac{1}{2}(\omega-1)^{-1}$, so gelation must occur at, or before, this time. (Note $M_{2}$ may or may not actually diverge at the gel point.) The exponent relation $\tau+\sigma=\omega+1$ should be valid in the pregelation stage. A tentative explanation of these paradoxical results is that solutions exist up to $t_{c}$, where gelation takes place, as $S_{1}\left(t_{c}\right)=\sum k s_{k} c_{k}\left(t_{c}\right)$ diverges. However, if at the same time the lower moment $S_{0}\left(t_{c}\right)$ diverges, then the kinetic equation (6.1) is no longer well defined beyond $t_{c}$, since it contains $S_{0}$. Our inequalities for $S_{0}(t)$ are, however, not sharp enough to confirm or exclude that $S_{0}\left(t_{c}\right)$ is indeed divergent. In any case, the coagulation equation with a sum kernel, having $\omega>1$, is physically unacceptable, unless (6.1) can be modified in such a manner that $S_{0}$ becomes finite again past $t_{c}$ by taking into account proper interactions between finite size clusters and infinite clusters. If one tries to make a Taylor expansion of $c_{k}(t)$ around $t=0$, additional difficulties are met for the case $\omega>1$, of the same nature as discussed in Section 5.4.

For the case $s_{k} \leqslant C k(k \rightarrow \infty)$ our conclusion is in agreement with the exact results of White, who showed that all moments remain finite on bounded time intervals, so that $M_{1}(t)=1$ for all $t>0$. The model with $K_{i j}=i^{\omega}+j^{\omega}$ and $\omega<1$ can be solved sequentially (see Appendix B). It has been used by Ruckenstein and Pulvermacher ${ }^{(27)}$ with $\omega=2 / 3$ to describe sintering-controlled catalyst aging.

A similar method may be applied to kernels of the more general form $K_{i j}=i^{\lambda} j^{\mu}+i^{\mu} j^{\lambda}$. For this case existence of global solutions has been proved by Leyvraz and Tschudi ${ }^{(3)}$ for $0<\mu \leqslant \lambda \leqslant 1$, where $K_{i j} \leqslant 2(i j)^{\max (\lambda, \mu)}$. If $\lambda, \mu \leqslant 1 / 2$, global solutions exist and gelation is absent according to White, ${ }^{(14)}$ since $i^{\lambda} j^{\mu}<i+j$. Now consider $\lambda>1 / 2$ and $\lambda \geqslant \mu$. 
Again we investigate the possibility of a postgelation solution to the coagulation equation:

$$
\dot{c}_{k}=\sum_{i+j=k} i^{\lambda} j^{\mu} c_{i} c_{j}-k^{\lambda} c_{k} M_{\mu}-k^{\mu} c_{k} M_{\lambda}
$$

Here we introduce two generating functions $f_{v}(x, t)(\nu=\lambda, \mu)$ as

$$
f_{v}(x, t)=\sum_{k=1}^{\infty} k^{v} c_{k} e^{k x} \simeq M_{\nu}+a_{\nu}(-x)^{\alpha_{\nu}}
$$

with $x \uparrow 0$ and $\alpha_{\nu}>0$, where the second equality represents the small- $x$ behavior. Using $\dot{M}_{0}=-M_{\lambda} M_{\mu}$ we deduce from (6.3)

$$
\dot{g}-\dot{M}_{0}=\left(f_{\lambda}-M_{\lambda}\right)\left(f_{\mu}-M_{\mu}\right)
$$

If (6.3) allows solutions with $\dot{M}_{1} \neq 0$, then (6.5) yields for small $x$

$$
\dot{M}_{1} x=a_{\lambda} a_{\mu}(-x)^{\alpha_{\lambda}+\alpha_{\mu}}
$$

Consequently

$$
\alpha_{\lambda}+\alpha_{\mu}=1, \quad a_{\lambda} a_{\mu}=-\dot{M}_{1}
$$

A small- $x$ singularity as in (6.4) yields according to (A.4) a large- $k$ behavior of $c_{k}$ of the form

$$
c_{k} \simeq \frac{a_{\lambda}}{\Gamma\left(-\alpha_{\lambda}\right) k^{\alpha_{\lambda}+1+\lambda}}=\frac{a_{\mu}}{\Gamma\left(-\alpha_{\mu}\right) k^{\alpha_{\mu}+1+\mu}}
$$

so that

$$
\begin{aligned}
& \alpha_{\mu}=\frac{1}{2}[1+\lambda-\mu] \equiv \frac{1}{2}+\alpha, \quad \alpha_{\lambda}=\frac{1}{2}-\alpha \\
& a_{\mu}=a_{\lambda} \Gamma\left(-\alpha-\frac{1}{2}\right) / \Gamma\left(\alpha-\frac{1}{2}\right)
\end{aligned}
$$

This yields finally

$$
c_{k} \simeq\left[\dot{M}_{1}\left(4 \alpha^{2}-1\right)(\cos \pi \alpha) / 4 \pi\right]^{1 / 2} k^{-[3+\lambda+\mu] / 2}
$$

First observe: if $\mu \leqslant 0$, then $\alpha_{\mu}=1$ in (6.4), since $a_{\mu} \sim \sum k^{1-|\mu|} c_{k} \leqslant M_{1}<\infty$ and (6.6) cannot be satisfied (for $\mu=0$ this is consistent with our conclusions for $K_{i j}=s_{i}+s_{j}$ ). Therefore $\mu>0$. Furthermore, if $\mu \leqslant \lambda-1$, then also $\alpha_{\mu}=1$ in (6.4), since $a_{\mu} \sim \sum k^{\mu+1} c_{k}<M_{\lambda}<\infty$, and (6.6) cannot be satisfied. Therefore $\mu>\lambda-1$. Finally the requirement $M_{1}<\infty$ imposes the condition $\lambda+\mu>1$. In summary: kinetic equations with kernels $K_{i j}$ $=i^{\lambda} j^{\mu}+i^{\mu} j^{\lambda}$ may allow solutions with a time-dependent total mass, provided

$$
\lambda, \mu>0, \quad|\mu-\lambda|<1, \quad \lambda+\mu>1
$$

For $|\lambda-\mu|>1$ and $\lambda, \mu>1$ this leads again to the same paradox of simultaneous occurrence of gelation, and absence of postgelation solutions, 
as discussed in the first part of this section. We expect again that the kinetic equation is only well-defined up to $t_{c}$.

If the conditions (6.11) are met and postgelation solutions exist, they have the form (1.3) with $\tau=\frac{1}{2}(\lambda+\mu+3$ ), whereas in the pregelation stage the exponent relation $\sigma+\tau=\lambda+\mu+1$ is valid.

For $\lambda>1$ or $\mu>1$ there exist again difficulties in the Taylor series expansion of $c_{k}(t)$ around $t=0$. One shows in a similar way that the kernels $K_{i j}=r_{i} s_{j}+s_{i} r_{j}$ with $r_{k} \sim k^{\omega}$ and $s_{k} \sim e^{\alpha k}$ for large $k$ and some (positive or negative) value of $\alpha$ do not admit postgelation solutions.

This result is consistent with the requirement $|\mu-\lambda|<1$ in (6.11), that the large- $k$ behavior of $r_{k}$ and $s_{k}$ not be too different.

For the kernel $K_{i j}=(i j)^{\omega}$ White ${ }^{(12)}$ has considered a steady state coagulation problem with a constant monodisperse source. In this case $\dot{c}_{k}$ in the coagulation equation is replaced by $-a \delta_{k 1}$ ( $a$ is the strength of the source), and the physical requirement, $M_{1}<\infty$, can be dropped.

The method of this section directly allows us to extend his results to both types of kernels discussed in this section. We then obtain asymptotic solutions, similar to (6.10) with $\dot{M}_{1}$ replaced by $-a$. The restriction (6.11) is relaxed to $|\lambda-\mu|<1$. This result applies also to the kernel $K_{i j}=i^{\omega}+j^{\omega}$, where $\lambda=0$ and $\mu=\omega<1$. For the latter kernel with $\omega=1$ White ${ }^{(12)}$ has already shown that no nontrivial solutions exist.

\section{CONCLUSION}

In this paper we have discussed the coagulation equations of chemical kinetics with different coagulation kinetics $K_{i j}$. We investigated the possibility of a gelation transition; different types of pre- and postgelation solutions; the validity of scaling; we calculated critical exponents as functions of the model parameters, and obtained bounds on the gel point $t_{c}$.

The model with coagulation kernel $K_{i j} \sim(i j)^{\omega}$ and $1 / 2<\omega \leqslant 1$ is of particular interest, ${ }^{(1,4)}$ as it effectively models the step-polymerization of $f$-functional monomeric units, in which a gelation transition occurs, and we compare our results with those of lattice percolation models.

An alternative to the chemical-kinetic theory of polymerization are lattice percolation models, where polymers are "grown" by randomly placing bonds between atoms on an appropriate lattice. ${ }^{(15-18)}$ Since such a model allows intermolecular reactions and also takes steric hindrance into account, it is believed to describe the properties about the gel point more accurately than the classical Flory-Stockmayer (FS) theory does. The two theories belong to a different universality class. In the FS theory a $k$-mercomposed of $k, f$-functional units-contains exactly $p_{k}=(k-1)$ bonds and $s_{k}=(f-2) k+2$ free ends, as a consequence of the assumption that only 
treelike structures can form with no intramolecular bonding (percolation on a Bethe lattice with coordination number $f$ ). In the reaction process all $s_{k}$ free ends are assumed to be equally reactive. In the kinetic formulation of the FS theory ${ }^{(5-9)}$ one deduces that the rate of formation of $(i+j)$-mers is proportional to $K_{i j}=s_{i} s_{j}$, where $K_{i j} \sim f^{2} i j$ for large $i, j$. Hence the reaction rate of a $k$-mer is proportional to its volume, $s_{k} \sim k$. We have modified this theory by assuming that the reaction of a large cluster is proportional to the number of reactive sites available for bonding between large clusters. ${ }^{14}$ Thus the coagulation equation with the kinetic $K_{i j}=s_{i} s_{j}$ can be used as a kinetic description of gelation and accounts for effects of cross-linking (with a polymer) and steric hindrance in an approximate manner.

We expect $s_{k}$ to have an approximate asymptotic behavior of the form $s_{k} \sim$ const $k^{\omega}(k \rightarrow \infty)$, described by the geometric exponent $\omega$, lying in the range $1-1 / d \leqslant \omega \leqslant 1$. The lower limit corresponds to the surface area of a compact solid; the upper limit represents the classical FS theory with complete reactivity. The exponent $\omega$ is a coarse-grained measure for the volume dependence of the effective surface area of a larger polymer ( $\omega d$ is its fractional dimension). This is reminiscent of the older Fisher droplet model, ${ }^{(28)}$ where an effective surface area with $\omega \simeq 0.8^{15}$ is considered. This effective surface should not be identified with the fine-grained external perimeter, which penetrates the whole cluster, ${ }^{(29)}$ and is therefore proportional to $k$. To obtain an estimate of $\omega$ we consider the mean radius of gyration $R_{k} \sim k^{\rho}$ as $k \rightarrow \infty$, ${ }^{(16)}$ which measures the linear dimension of a cluster, and thus $\omega \simeq(d-1) \rho$. Past the gel point clusters are compact with $\rho_{>}=1 / d$ and $\omega=1-1 / d$. At the gel point they are more dilute, since $\rho_{c} \simeq 0.53$ (2D) and $\rho_{c} \simeq 0.40$ (3D). A finite time before the gel point $\rho_{<} \simeq 0.641$ (2D) and $\rho_{<} \simeq 0.5$ (3D), so that these clusters (lattice animals) have an even more dilute structure.

What are the predictions of this model? Scaling holds for $t<t_{c}$, as shown by Leyvraz and Tschudi, ${ }^{(4)}$ but not for $t>t_{c}$ due to the absence of sol-gel interactions in the coagulation equations (see Section 2.3). For the exponents we have in (1.4) and (5.27), respectively, $\tau^{\prime}=\omega+3 / 2\left(t \geqslant t_{c}\right)$ and $\sigma+\tau=2 \omega+1\left(t<t_{c}\right)$. Assuming $\tau=\tau^{\prime}$, we find the nonclassical exponents: $\tau^{\prime}=\tau=\omega+3 / 2$ and $\sigma=\omega-1 / 2 ; \gamma$ is given by (1.13) and $\beta=1$ (classical value), as shown in Section 2.3. In Table III we have compared the kinetic values for $\tau$ and $\sigma$ for dimensions $d=2,3, \ldots, 6$ with those obtained from lattice models, assuming compact clusters. The kinetic values fall slightly below the lattice results if $\omega \simeq 1-1 / d$. For the

\footnotetext{
${ }^{14}$ A small cluster may penetrate deep inside a large cluster before it is bounded, and its reaction rate is not proportional to $s_{k}$.

${ }^{15} \omega=(d-1) \rho_{c}$ (see below).
} 
Table III. The Kinetic Theory; Values of the Exponents $\tau$ and $\sigma$ for Compact Clusters with $\omega=1-1 / d$, Compared with those Obtained in Lattice

Percolation Models. ${ }^{a}$

\begin{tabular}{ccccc}
\hline$d$ & $\tau=\omega+3 / 2$ & $\tau$ (lattice) & $\sigma=\omega-1 / 2$ & $\sigma$ (lattice) \\
\hline 2 & 2.00 & 2.06 & 0.00 & 0.40 \\
3 & 2.17 & 2.20 & 0.17 & 0.46 \\
4 & 2.25 & 2.30 & 0.25 & 0.5 \\
5 & 2.30 & 2.39 & 0.30 & 0.5 \\
6 & 2.33 & 2.50 & 0.33 & 0.5 \\
\hline
\end{tabular}

${ }^{a}$ Lattice values are deduced from Refs. 15 and 16 using scaling relations.

exponent $\sigma$ the disagreement is significant if $\omega \simeq(d-1) \rho_{>}$and somewhat smaller for $\omega \simeq(d-1) \rho_{c}$ (see Refs. 15 and 16). Finally we obtained a bound (5.26) for the gel-point, which is (for monodisperse initial conditions) $t_{c} \geqslant\left\{2^{2 \omega-1}-1\right\}^{-1}$.

This kinetic model belongs to a universality class neither of the lattice percolation models nor of the classical FS theory. This is also true for kinetic models of gelation, recently studied by computer simulation. ${ }^{(19,30)}$ The model in Ref. 19 yields $\gamma=2.0$. Our model yields $\gamma=5$ for compact clusters $\left(\omega=2 \rho_{>}=2 / 3\right)$ and $\gamma=2.3$ for percolating clusters $\left(\omega=2 \rho_{c} \cong\right.$ 0.8 ), suggesting that the two models fall in different universality classes.

A disadvantage of the kinetic approach in describing realistic systems is that the coagulation rates $K_{i j}$, used in the extensive literature on the kinetics of polymerization, ${ }^{(1,3,4,6-14)}$ only account for the statistical probability of bond formation, the effect of diffusion being neglected. The limitations on the reaction rates, imposed by diffusion, have been studied in connection with colloidal and aerosol coagulation, ${ }^{(2)}$ but very little has been done in the context of polymerization and gelation. Noyes ${ }^{(35)}$ has given a general discussion of the effects of diffusion in chemical kinetics. He calculated the effective (steady) rate constant, which for the coagulation rate of an $i$ and $j$ cluster becomes

$$
\begin{aligned}
K_{i j}^{*} & =K_{i j}\left\{1+K_{i j} / B_{i j}\right\}^{-1} \\
B_{i j} & =4 \pi \cdot\left(D_{i}+D_{j}\right)\left(R_{i}+R_{j}\right)
\end{aligned}
$$

Here $K_{i j}$ is the statistical reaction rate, $D_{i}+D_{j}$ is the diffusion coefficient for relative motion of an $i$ and a $j$ cluster $^{(32)} ; R_{k}$ is the radius of gyration of a $k$-cluster and $B_{i j}$ the coagulation rate for Brownian coagulation. ${ }^{(32)}$

The kinetic approximation $K_{i j}^{*} \simeq K_{i j}$ is valid when $K_{i j} \ll B_{i j}$ holds, i.e., in the limit of infinite diffusivity. In the opposite case $K_{i j} \ll B_{i j}$ the effective rate constants are $K_{i j}^{*} \simeq B_{i j}$ and the reaction is said to be controlled by 
diffusion. Which form of $K_{i j}$ is applicable depends on the values of $R_{k}, D_{k}$, and $K_{i j}$. The diffusivity of a Brownian particle $D_{k} \sim\left(\eta R_{k}\right)^{-1}$ depends on the viscosity $\eta$ of the solvent, on the cluster radius $R_{k}$, and on the boundary conditions imposed at the surface of the particles. Both the diffusivity $D_{k}$ and the radius of gyration $R_{k}$ for lattice clusters in different regimes $\left(t<t_{c}\right.$, $t=t_{c}, t>t_{c}$ ) can be found in a paper by Gould and Hall, ${ }^{(31)}$ who list values for the exponents $\rho$ and $y$, defined through $R_{k} \sim k^{\rho}$ and $D_{k} \sim k^{-y}$ $(k \rightarrow \infty)$. These exponents assume different values before, at, and after $t_{c}$. For very large clusters of approximate size $L$ the kinetic coagulation kernel $K_{i j}=(i j)^{\omega}$, as used in the present paper, is of order $L^{2 \omega}$ and much larger than $B_{i j}$. For compact clusters ${ }^{(31)}$ and Brownian particles of size $L$, $B_{i j} \sim$ const; for lattice clusters $B_{i j} \sim L^{-y}<$ const $L^{1 / 2}$. In the large- $L$ regime the reaction is thus controlled by diffusion, $K_{i j}^{*} \simeq B_{i j}$. This kernel can be bounded from above by $C(i+j)$ and the solution of the coagulation equation will not show a gelation transition. However, the kinetic approach to gelation will be useful if there exists a sufficiently large regime $i, j<L$ where $K_{i j}^{*} \simeq K_{i j}$. Then there will be two regimes in the large- $k$ behavior of the clusters for $t>t_{c}$. For $1 \ll k<L$ the size distribution will show an algebraic decay $\sim k^{-\tau}$ which gradually changes into exponential decay $C_{k} \sim k^{-\tau} \exp (-$ const $k)$ for $k \gg L$. The total mass $M_{1}(t)=\sum_{k=1}^{\infty} c_{k}(t)$ is strictly conserved when diffusion is taken into account, but the quantity $M_{1, L}(t) \equiv \sum_{k=1}^{L} k c_{k}(t)$ does behave approximately as the quantity $M_{1}(t)$ in our model where $K_{i j}=(i j)^{\omega}$ for all large $i, j$. Stated differently, clusters in the actual polymerizing system in a good solvent with a size much larger than $L$ are essentially interpreted as infinite, i.e., belonging to the gel.

An alternative veiw ${ }^{(12)}$ could be that the kinetic equation (1.1) describes coagulation processes in a continuously stirred container (corresponding formally to infinitely high diffusities).

After this application of the general theory to a special problem, we make some comments concerning our general results:

1. We have not given any rigorous proofs, but tentatively assumed that in all cases the solution to the initial-value problem exists and is unique. Rigorous proofs exists for $K_{i j} \leqslant i j(i, j \rightarrow \infty)$ but for kernels $K_{i j}>i j$ $(i, j \rightarrow \infty)$ more theorems concerning existence and uniqueness are desired [in view of some difficulties (see Section 5.4) the the Taylor series expansion of $c_{k}(t)$ around $\left.t=0\right]$, as well as a rigorous proof that the limiting form (5.4) of the moment equations (on which many conclusions were based) is valid throughout the entire pregelation stage.

2. Very recently Leyvraz and Tschudi ${ }^{(4)}$ have considered the same model $K_{i j}=(i j)^{\omega}$ with $0<\omega<1$. They found a number of results coinciding with ours. These are: the exponent relation $\tau=\omega+3 / 2$ and $\sigma=\omega-$ $1 / 2$; the absence of gelation for $\omega \leqslant 1 / 2$ (theorem), the occurrence of gelation for $\omega>1 / 2$ (conjecture), and the discovery of the special postgela- 
tion solution (1.15). Their method is, however, slightly different. In the postgelation stage these authors determine the exponent $\tau$ by inserting the ansatz $c_{k}(t) \simeq C k^{-\tau}$ into the coagulation equation, from which the exponent $\tau=\omega+3 / 2$ and the amplitude $C$ in (2.10) follow, while in our method the small- $x$ behavior (2.8) of the generating function is determined. In the pregelation stage Leyvraz and Tschudi give arguments in favor of scaling, essentially by substituting the ansatz $c_{k} \simeq k^{-\tau} \Phi(k F(t))$ ( $k$ large, $t_{c}-t$ small) into the coagulation equation. Consistency then requires that $F(t)$ $\sim\left(t_{c}-t\right)^{1 / \sigma}$ with $\sigma=\omega-1 / 2$. Leyvraz and Tschudi have the additional result $\Phi(x) \sim \exp \left(-C x^{\omega}\right)$ for $x \rightarrow 0$. We show that the coagulation equation allows similarity solutions of the form $c_{k}(t) \simeq k^{-\tau} \Phi\left(k\left(t_{c}-t\right)^{1 / \sigma}\right)(k \rightarrow \infty)$, provided the exponents are related as $\tau+\sigma=2 \omega+1$. The absence of gelation for $\omega \leqslant 1 / 2$ in our method follows from an inequality for the second moment, while our arguments ${ }^{(1)}$ and those in Ref. 4 for the occurrence of gelation and the validity of the special postgelation solution for $\omega>1 / 2$ are essentially the same. In addition we have for the cases $\omega=2$ and $\omega \gg 1$ determined the special postgelation solution analytically, and for the cases $1 / 2<\omega<10$ numerically.

3. We intend to support and expand these results by analyzing numerical solutions to the coagulation equation in order to test the validity of scaling, predicted exponents, estimates for $t_{c}$, etc.

4. Extensions of the theory can be made in several directions. It would be of interest to modify the coagulation equation past the gel point by including proper interactions between sol and gel (see Section 2.3), such as in Flory's theory of gelation ${ }^{(4)}$ for the classical case where $K_{i j} \sim i j$, as discussed by Ziff and Stell. ${ }^{(9)}$ The most important questions are then whether proper modifications can be found such that the scaling property also holds past the gel point, and whether the postgelation exponents will remain the same. The same questions should be asked for pre-and postgelation solutions in reversible polymerization processes, where fragmentation terms are added that would stop the reaction at a finite value $t$ before or after $t_{c}$.

\section{ACKNOWLEDGMENT}

R.M.Z. acknowledges the Office of Basic Energy Sciences, U.S. Department of Energy, for support of this research.

\section{APPENDIX A}

Here we show how singularities in the generating function (2.2), for small negative $x$, are related to the asymptotic behavior of the size distribution, $c_{k}$, for large $k$. 
The Bose-Einstein functions

$$
g_{a}(x) \equiv \sum_{k=1}^{\infty} k^{-\alpha} e^{k x}=[\Gamma(\alpha)]^{-1} \int_{0}^{\infty} d y y^{\alpha-1}\left\{e^{y-x}-1\right\}^{-1}
$$

have the representation ${ }^{(33,34)}$

$$
g_{\alpha}(x)=\left\{\begin{array}{rr}
\Gamma(1-\alpha)(-x)^{\alpha-1}+\sum_{k=0}^{\infty} \zeta(\alpha-k) x^{k} / k ! & \\
& (\alpha \neq 1,2, \ldots) \quad(\text { A. } 2) \\
{[\Gamma(\alpha)]^{-1} x^{\alpha-1}[-\log (-x)+C+\Psi(\alpha)]+\sum_{k=0}^{\infty} \zeta(\alpha-k) x^{k} / k !} & (\alpha=1,2, \ldots)
\end{array}\right.
$$

where the prime on the sum indicates that the term with $k=\alpha-1$ is to be omitted. Here $\zeta(n)$ is the Riemann zeta function, $\psi(x)=\Gamma^{\prime}(x) / \Gamma(x)$, and $C$ is Euler's constant.

In both cases above the first term represents the singularity of the function, the remaining sum being an entire function. From (A.2), we see that if $g(x) \equiv \sum c_{k} e^{x k}$ has a singularity $(-x)^{\alpha}(\alpha \neq 1,2,3, \ldots)$ then $c_{k}$ has the following asymptotic behavior:

$$
g(x) \sim(-x)^{\alpha} \leftrightarrow c_{k}>k^{-\alpha-1} / \Gamma(-\alpha)
$$

Note that if $(-x)^{\alpha}$ were the only singularity in $g$ there would be no higher algebraic terms in addition to (A.4). For if $c_{k}$ had a term (say) $k^{-\alpha-2}$ in addition to (A.4), then by (A.2) $g$ would have an $(-x)^{\alpha+1}$ singularity.

If $g(x)$ has the singularity $-\log (-x) x^{\alpha}$, where $\alpha$ is an integer, then it follows from (A.3) that

$$
g(x) \sim-x^{\alpha} \log (-x) \leftrightarrow c_{k} \sim k^{-\alpha-1} \alpha !
$$

Finally, if $g(x)$ has singularities of the form

$$
-(-x)^{\alpha} \log (-x)=-\frac{d}{d \alpha}(-x)^{\alpha}
$$

with $\alpha \neq$ integer, then application of $d / d \alpha$ to (A.4) yields for $k \gg \alpha$

$$
g(x) \sim-(-x)^{\alpha} \log (-x) \leftrightarrow c_{k} \sim k^{-\alpha-1} \log k / \Gamma(-\alpha)
$$

\section{APPENDIX B}

In this appendix we show how the coagulation equation (1.1) with the kernel $K_{i j}=i^{\omega}+j^{\omega}$ and $\omega<1$

$$
\dot{c}_{k}=\sum_{i+j=k} i^{\omega} c_{i} c_{j}-k^{\omega} c_{k} M_{0}-c_{k} M_{\omega}
$$


can be solved sequentially by changing to new variables. First observe that the equation for $c_{1}$ can be integrated, i.e.,

$$
\begin{aligned}
\dot{c}_{1} & =-\left(M_{0}+M_{\omega}\right) c_{1} \\
\dot{M}_{0} & =-M_{0} M_{\omega}
\end{aligned}
$$

to yield for monodisperse initial conditions, $c_{k}(0)=\delta_{k 1}$ :

$$
c_{1}(t)=M_{0}(t) \exp \left[-\int_{0}^{t} d t^{\prime} M_{0}\left(t^{\prime}\right)\right]
$$

New variables $\nu_{k}$ and $\tau$ are introduced by the transformation

$$
\begin{aligned}
\tau & =\int_{0}^{t} d t^{\prime} M_{0}\left(t^{\prime}\right) \\
v_{k}(\tau) & =c_{k}(t) / M_{0}(t)
\end{aligned}
$$

The kinetic equation becomes

$$
\frac{d \nu_{k}(\tau)}{d \tau}=\sum_{i+j=k} i^{\omega} \nu_{i} \nu_{j}-k^{\omega} \nu_{k}
$$

to be solved subject to the condition $\sum v_{k}=1$, as follows from (B.4). The equation for $\dot{\nu}_{k}$ involves only $\nu_{l}$ with $l \leqslant k$, and the set can therefore be solved sequentially. Once all $\nu_{k}(\tau)$ have been determined, the function $M_{0}(t) \equiv \mu_{0}(\tau)$ can be found as a function of $\tau$ from

$$
\sum_{k} k \nu_{k}=1 / \mu_{0}(\tau)=1 / M_{0}(t)
$$

and the original $t$ variable can be recovered by differentiating $\tau(t)$ and solving for $t$, with the result

$$
\int_{0}^{\tau} \frac{d \tau^{\prime}}{\mu_{0}\left(\tau^{\prime}\right)}=t
$$

The solutions for the first three $\nu_{l}$, starting from a monodisperse initial distribution $\nu_{k}(0)=\delta_{k 1}$, explicitly read

$$
\begin{aligned}
\nu_{1}(\tau)= & e^{-\tau} \\
\nu_{2}(\tau)= & \left(2^{\omega}-2\right)^{-1}\left[\exp (-2 \tau)-\exp \left(-2^{\omega} \tau\right)\right] \\
\nu_{3}(\tau)= & -\left(2^{\omega}-2\right)^{-1}\left(3^{\omega}-2^{\omega}-1\right)^{-1}\left(2^{\omega}+1\right) \\
& \times\left\{\exp \left[-\left(2^{\omega}+1\right) \tau\right]-\exp \left(-3^{\omega} \tau\right)\right\} \\
& +\left(2^{\omega}-2\right)^{-1}\left(3^{\omega}-3\right)^{-1}\left(2^{\omega}+1\right)\left\{\exp (-3 \tau)-\exp \left(-3^{\omega} \tau\right)\right\}
\end{aligned}
$$

We have not been able to determine $v_{k}(t)$ for general $k$. It should be noted that this method cannot be used for $\omega>1$ without care, since the kinetic equation (B.1) is only well defined for $t \leqslant t_{c} \leqslant \frac{1}{2}(\omega-1)^{-1}$ [see the text 
below Eq. (6.2)]. Furthermore, $M_{0}(t)$ would become negative within a finite time $t_{0}$ [see (5.18)], so that there does not exist a one-to-one relationship between $t$ and $\tau$ in (B.4).

For the case $\omega=1$ the solution of (B.5) is well known. ${ }^{(2,10,11)}$ It is of interest to note that (B.5) can be written in terms of new variables $n_{k}(\tau)=\nu_{k}(\tau) / k$, using $\sum v_{k}=\sum k n_{k}=1$ as follows:

$$
\begin{aligned}
d n_{k} / d \tau & =k^{-1} \sum_{i+j=k} i(i j) n_{i} n_{j}-k n_{k} \\
& =\frac{1}{2} \sum_{i+j=k}(i j) n_{i} n_{j}-\sum_{j=1}^{\infty}(k j) n_{k} n_{j}
\end{aligned}
$$

This is just the coagulation equation (1.1) with $K_{i j}=i j$. Thus we have established a correspondence between the two (exactly soluble) models $K_{i j}=i+j$ and $K_{i j}=i j$, and their solutions are related. For example, for monodisperse initial conditions we find $\tau=1-e^{-t}$ and the corresponding solution of (B.1) can be found from (3.1) using the transformation

$$
\tilde{c}_{k}(t)=k c_{k}(\tau), \quad \tau=1-e^{-t}
$$

Note that $\tau=\tau_{c}=1$ is mapped onto $t=\infty$.

\section{REFERENCES}

1. R. M. Ziff, E. M. Hendricks, and M. H. Ernst, Phys. Rev. Lett. 49:593 (1982).

2. R. L. Drake, in Topics in Current Aerasol Research, Vol. 3, Pt. 2, G. M. Hidy and J. R. Brock, eds. (Pergamon Press, New York, 1972).

3. F. Leyvraz and H. R. Tschudi, J. Phys. A: Math. Gen. 14:3389 (1981).

4. F. Leyvraz and H. R. Tschudi, J. Phys. A: Math. Gen. 15:1951 (1982).

5. P. J. Flory, Principles of Polymer Chemistry (Cornell University, Ithaca, New York, 1953), Chap. 9.

6. W. H. Stockmayer, J. Chem. Phys. 11:45 (1943).

7. K. Dušek, Polymer Bull. 1:523 (1979).

8. R. M. Ziff, J. Stat. Phys. 23:241 (1980).

9. R. M. Ziff and G. Stell, J. Chem. Phys. 73:3492 (1980).

10. R. J. Cohen and G. B. Benedek, J. Phys. Chem. 86:3696 (1982); G. v. Schulthess, G. B. Benedek, and R. W. de Blois, Macromolecules 13:940 (1980).

11. A. Lushnikov, J. Colloid Interface Sci. $45: 549$ (1973); 48:400 (1974); $54: 94$ (1975).

12. W. White, J. Colloid Interface Sci. 87:204 (1982).

13. J. B. Mcleod, Quart. J. Math. 13:119 (1962); 13:192 (1962); 13:283 (1962).

14. W. White, Proc. Am. Math. Soc. 80:273 (1980).

15. D. Stauffer, Phys. Rep. 54:1 (1979).

16. D. Stauffer, A. Coniglio, and M. Adam, in Advances in Polymer Science, Vol. 44, p. 103, K. Dušek, ed. (Springer-Verlag, Berlin, 1982).

17. J. W. Essam, Rep. Progr. Phys. 43:833 (1980).

18. H. E. Stanley, P. J. Reynolds, S. Redner, and F. Family, in Real Space Renormalization, T. W. Burkhard and J. M. J. van Leeuwen, eds. (Springer-Verlag, Berlin, 1982), Chap. 7.

19. H. J. Herrmann, D. R. Landau, and D. Stauffer, Phys, Rev. Lett. 49:412 (1982). 
20. W. Schmidt and W. Burchard, Macromolecules 14:370 (1981).

21. M. Abramowitz and I. Stegun, Handbook of Mathematical Functions (Dover, New York, 1974), p. 14.

22. K. Rektorys, Survey of Applicable Mathematics (Iliffe Books, London, 1969), p. 1252.

23. J. Silk and S. D. White, Astrophys. J. 223:L59 (1978).

24. W. F. Ames, Nonlinear Ordinary Differential Equations in Transport Processes (Academic Press, New York, 1974).

25. G. W. Bluman and J. D. Cole, Similarity Methods for Differential Equations (Springer, New York, 1974).

26. M. H. Ernst, in Studies in Statistical Mechanics X, J. L. Lebowitz and E. W. Montroll, eds. (North-Holland, Amsterdam, 1982).

27. E. Ruckenstein and B. Pulvermacher, AIChE. J. 19:356 (1973).

28. M. E. Fisher, Physics 3:225 (1967); D. Stauffer, C. S. Kiang, and G. H. Walker, J. Stat. Phys. 3:323 (1971).

29. H. Franke, Z. Phys. B 45:247 (1981).

30. P. Manneville and L. de Seze, in Numerical Methods in the Study of Critical Phenomena, I. Della Dora, J. Demongeot, and B. Lacolle, eds. (Springer, Berlin, 1981).

31. H. Gould and Holl, J. Phys. A 14:L443 (1981).

32. S. K. Friedlander, Smoke, Dust and Haze (Wiley, New York, 1977).

33. J. E. Robinson, Phys. Rev. 83:678 (1951).

34. R. M. Ziff, Phys. Rep. 32:216 (1977).

35. R. M. Noyes, in Progress in Reaction Kinetics I, G. Porter, ed. (Pergamon Press, Oxford, 1961), Chap. 5.

36. J. D. Klett, J. Atmosph. Sci. 32:380 (1975). 